\title{
Early Sowing Combined with Adequate Potassium and Sulfur Fertilization: Promoting Beta vulgaris (L.) Yield, Yield Quality, and K- and S-Use Efficiency in a Dry Saline Environment
}

\author{
Ali A. A. Mekdad ${ }^{1}\left(\mathbb{D},{\text { Mostafa M. } \operatorname{Rady}^{2, *} \mathbb{D}, \text { Esmat F. Ali }^{3}(\mathbb{D}) \text { and Fahmy A. S. Hassan }}^{3}(\mathbb{D})\right.$ \\ 1 Agronomy Department, Faculty of Agriculture, Fayoum University, Fayoum 63514, Egypt; \\ aam07@fayoum.edu.eg \\ 2 Botany Department, Faculty of Agriculture, Fayoum University, Fayoum 63514, Egypt \\ 3 Department of Biology, College of Science, Taif University, P.O. Box 11099, Taif 21944, Saudi Arabia; \\ a.esmat@tu.edu.sa (E.F.A.); d.fahmy@tu.edu.sa (F.A.S.H.) \\ * Correspondence: mmr02@fayoum.edu.eg; Tel.: +2-084-010-923-920-38
}

Citation: Mekdad, A.A.A.; Rady, M.M.; Ali, E.F.; Hassan, F.A.S. Early Sowing Combined with Adequate Potassium and Sulfur Fertilization: Promoting Beta vulgaris (L.) Yield, Yield Quality, and K- and S-Use Efficiency in a Dry Saline Environment. Agronomy 2021, 11, 806. https://doi.org/10.3390/ agronomy11040806

Received: 1 March 2021

Accepted: 16 April 2021

Published: 19 April 2021

Publisher's Note: MDPI stays neutral with regard to jurisdictional claims in published maps and institutional affiliations.

Copyright: (c) 2021 by the authors. Licensee MDPI, Basel, Switzerland. This article is an open access article distributed under the terms and conditions of the Creative Commons Attribution (CC BY) license (https:// creativecommons.org/licenses/by/ $4.0 /)$.

\begin{abstract}
Field trials for two seasons (2018/2019 and 2019/2020) were conducted to investigate the influence of the addition of three levels of potassium $(\mathrm{K})\left(\mathrm{K}_{1}=60, \mathrm{~K}_{2}=120\right.$, and $\mathrm{K}_{3}=180 \mathrm{~kg}$ $\mathrm{K}_{2} \mathrm{O}$ ha $\left.{ }^{-1}\right)$ and/or sulfur $(\mathrm{S})\left(\mathrm{S}_{1}=175, \mathrm{~S}_{2}=350\right.$, and $\left.\mathrm{S}_{3}=525 \mathrm{~kg} \mathrm{CaSO}_{4} \mathrm{ha}^{-1}\right)$ to the soil, as well as the sowing date (the 1 st of September, $\mathrm{D}_{1}$; or the 1 st of October, $\mathrm{D}_{2}$ ) on the potential improvement of physiology, growth, and yield, as well as the quality characteristics of sugar beet yield under soil salinity conditions. With three replicates specified for each treatment, each trial was planned according to a split-split plot in a randomized complete block design. The results revealed that early sowing $\left(\mathrm{D}_{1}\right)$ led to significant improvements in all traits of plant physiology and growth, in addition to root, top, and biological yields and their quality, gross and pure sugar, and K- and S-use efficiencies based on root yield (R-KUE and R-SUE). The $\mathrm{K}_{3}$ level $\left(180 \mathrm{~kg} \mathrm{~K}_{2} \mathrm{O}\right.$ ha ${ }^{-1}$ ) positively affected the traits of plant physiology, growth, yield and quality, and R-SUE, and reduced the attributes of impurities, impurity index, and R-KUE. Additionally, the $\mathrm{S}_{3}$ level $\left(525 \mathrm{~kg} \mathrm{CaSO}_{4} \mathrm{ha}^{-1}\right)$ affirmatively affected plant physiology, growth, yield and quality traits, and R-KUE, and decreased impurity traits, impurity index, and R-SUE. The interaction of $\mathrm{D}_{1} \times \mathrm{K}_{3} \times \mathrm{S}_{3}$ maximized the yield of roots (104-105 ton ha ${ }^{-1}$ ) and pure sugar (21-22 ton ha ${ }^{-1}$ ). Path coefficient analysis showed that root yield and pure sugar content had positive direct effects with 0.62 and 0.65 , and 0.38 and 0.38 in both studied seasons, respectively, on pure sugar yield. Significant $(p \leq 0.01)$ positive correlations were found between pure sugar yield and root yield $\left(\mathrm{r}=0.966^{* *}\right.$ and $\left.0.958^{* *}\right)$. The study results recommend the use of the integrative $\mathrm{D}_{1} \times \mathrm{K}_{3} \times \mathrm{S}_{3}$ treatment for sugar beet to obtain maximum yields and qualities under salt stress (e.g., $8.96 \mathrm{dS} \mathrm{m}^{-1}$ ) in dry environments.
\end{abstract}

Keywords: sowing date; potassium use efficiency; sulfur use efficiency; path coefficient; correlations and stepwise regression

\section{Introduction}

During the period of growth to maturity, plants face many environmental stresses, including salinity, especially in arid and semiarid environments in most parts of the world, including Egypt, where soil salinity increases annually; therefore, these adverse conditions cause a major ecological problem and restrict the performance of plants [1-5]. Salinity is the biggest enemy for plants because, as harmful environmental stress, it causes another harmful stress, "osmotic stress", which is the lack of water [6,7].

Soil salinity is associated with many factors that destroy the productivity of different crop plants such as low fertility, poor structure, and water restrictions or "osmotic stress" in many parts of the world [8,9]. These destructive factors cause changes in plant metabolism and key physio-biochemical and molecular processes and ultimately reduce 
plant growth, then yield, and quality, especially in dry regions. The decrease in crop growth and production due to soil salinity is attributed to ion toxicity, nutritional imbalance, reduced enzymatic and osmotic effects, decreased photosynthetic efficiency, as well as more negative physiological changes $[3,4,9,10]$.

As for sugar production, sugar beet (Beta vulgaris L.) is the second source of sugar in many countries of the world, including Egypt. Like many countries around the world, the Egyptian agricultural policy encourages increasing the cultivated areas of sugar beet to increase the production of pure sugar to minimize the gap between sugar consumption and production, as well as meet the requirements of modern established factories. Like other economical crops, sugar beet requires a suitable sowing date and an appropriate integrative fertilization program to become an effective productive crop, especially given the above-mentioned environmental constraints.

Under dry environmental conditions, the proper sowing date of sugar beet plays a vital role in growth, production, physiological, and quality traits. It has been reported that the early sowing of sugar beet causes a marked increase in gross sugar content, and the highest yields of roots and sugars [11-13]. The key environmental variables that define the start of beet growing are temperature, soil moisture, and slight rate of rainfall [14]. As previously mentioned, sowing sugar beet during October markedly increases root weight and yield, total sugar content, and gross sugar yield compared to sowing in November [15]. Another recent study displayed that the early sowing of sugar beet improves quality traits, i.e., sucrose content and quality index. Conversely, the traits of the impurities (e.g., $\mathrm{K}, \mathrm{Na}$, and $\alpha$ - amino $N$ ) are varied [13].

Beyond the temperature factor (sowing date), some basic factors in salt-affected soils negatively affect the growth and yields of sugar beet such as low soil fertility, the unavailability of key nutrients (e.g., $\mathrm{K}, \mathrm{S}$, and $\mathrm{Mg}$ ), increased $\mathrm{Na}^{+}$ion, and limited irrigation water, as well as unbalanced fertilization of $\mathrm{N}, \mathrm{P}$, and $\mathrm{K}[16,17]$. To increase crop plant tolerance to soil salinity by mitigating $\mathrm{Na}^{+}$and $\mathrm{Cl}^{-}$damage [18], studies have shown that applying $\mathrm{K}$ mitigates the damaging effects of soil salinity on plant growth and productivity $[3,19]$. It has been confirmed that the enhancing impact of $\mathrm{K}$ in increasing sugar beet salt tolerance is achieved through enhancing plant nutritional status and increasing biosynthesis of organic metabolites [20]. As a regulator of plant water content, $\mathrm{K}$ is essential for the growth and production of crop plants due to its major role in protein synthesis, osmoregulation, enzyme activation, stomatal movement, photosynthesis, cation-anion homeostasis, and phloem transport [21,22], as well as increasing salinity tolerance in sugar beet [23]. The use of $\mathrm{K}$ fertilizer decreases salt stress effects and increases root, top, and sugar yields, pure and gross sugar content, sugar yield, and juice quality characteristics [24-26].

In arid environments, including Egypt, salt-affected soils suffer from high $\mathrm{pH}$ and the unavailability of essential nutrients, including $\mathrm{K}$ and $\mathrm{S}$, with high contents of $\mathrm{Na}^{+}$and $\mathrm{Cl}^{-}$, as well as low fertility and poor structure $[27,28]$. For such soils, the application of sulfur (S) may assist in eliminating soil alkalinity during biological $\mathrm{S}$ oxidation, and it could enhance crop yield and quality. It is essential for the desired plant growth because it is a component of many key coenzymes and amino acids, such as methionine and cysteine, which are required for the production of structural proteins, and is concerned with the synthesis of chlorophyll, some vitamins, proteins, and carbohydrates [29,30]. Dry environment soils, including Egypt, are becoming deficient in the nutrient $S$, and thus the use of $S$ as free fertilization is vital to enhance crop yields, including root and sugar yields [31]. $\mathrm{S}$ deficiency leads to changes in protein synthesis and may affect the assimilates, thus reducing sugar transformation and storage in sugar beet tubers due to a deficiency in foliar chlorophyll [32]. Application of S at $25 \mathrm{~kg} \mathrm{ha}^{-1}$ results in a $25 \%$ increase in root yield, as well as improved root quality traits through a decrease in $\alpha$-amino N [33]. Moreover, several positive effects related to sugar beet growth, production, and quality characteristics have been demonstrated [34,35].

However, research work discussing the investigation of potential improvements with early sowing date in combination with $\mathrm{K}$ and/or S applied as soil supplementation has 
not yet been investigated with sugar beet plants growing in saline soil located in semi-arid environments. Therefore, the current study hypothesized that an early sowing date (the 1st of September compared to the 1st of October) in combination with $\mathrm{K}(60,120$, or $180 \mathrm{~kg}$ $\left.\mathrm{K}_{2} \mathrm{O} \mathrm{ha}^{-1}\right)$ and/or $\mathrm{S}\left(175,350\right.$, or $525 \mathrm{~kg} \mathrm{CaSO}_{4} \mathrm{ha}^{-1}$ ) applied as soil supplementations may mitigate the osmotic stress and ionic imbalance induced by salinity through the positive influences on morpho-physiological traits, yield components, and yield quality, as well as $\mathrm{K}$ - and S-use efficiency (based on root yield) in sugar beet grown under soil salinity $\left(\mathrm{ECe}=8.96 \mathrm{dS} \mathrm{m}^{-1}\right)$ conditions over two seasons in an arid environment.

\section{Materials and Methods}

\subsection{Field Trials Details}

Two consecutive field experiments, at two different locations on the same site, were carried out for the 2018/2019 and 2019/2020 winter seasons at an experimental station $\left(29^{\circ} 17^{\prime} \mathrm{N}, 30^{\circ} 53^{\prime} \mathrm{E}\right.$, Southeast Fayoum) located at the Faculty of Agriculture experimental farm, Fayoum University. Each experiment was arranged in a split-split plot (three factorssowing dates, potassium fertilization, and sulfur fertilization-were applied in main plots, subplots, and sub-subplots, respectively) in a randomized complete block design (RCBD), and each experimental treatment was repeated three times. The size of the basic experimental unit was $10.5 \mathrm{~m}^{2}$, consisting of 5 rows of $3.5 \mathrm{~m}$ in length and $60 \mathrm{~cm}$ in width (i.e., row spacing).

The experimental region is climatically classified as semi-arid [36] on the aridity scale. Pre-season physicochemical characteristics [37] of the $0-50 \mathrm{~cm}$ soil depth from the 2018/19 and 2019/20 seasons are presented in Table 1. The experimental soil samples were classified as sandy loam [38] by the USDA Soil Taxonomy. Soil samples were air-dried and sieved through a 2-mm sieve. Approximately $300 \mathrm{~g}$ of soil for ECe measurement was dried, ground, passed through a 10-mesh screen, and saturated with distilled water for $24 \mathrm{~h}$. The $\mathrm{pH}$ values of soil samples were measured in saturated soil-water paste using a Bekman pH meter (model Elico, LI120-UK) [37]. Several milliliters of soil-water paste were extracted through a Whitman No. 1 paper filter in Buchner funnel with a vacuum system. The electrical conductivity $\left(\mathrm{EC} 25^{\circ} \mathrm{C}\right)$ of the soil-paste extracts was determined using a calibrated, temperature-compensating, digital readout conductivity instrument (model 3200, YSI, Inc., Yellow Springs, OH, USA) [37].

Healthy seeds of sugar beet (var. BTS 301 multigerm, Germany; Moderately Tolerant to Salt) were obtained from the Sugar Crops Research Institute, Egyptian Agricultural Research Center, and the seeds were sown on the 1st of September and October in each of the first and second seasons. After sterilization with $1 \%(v / v)$ sodium hypochlorite, $2-4$ sugar beet seeds were sown in each hill $20 \mathrm{~cm}$ apart. Thirty days after planting (DAS) (4-6 leaf stage), the seedlings were thinned to one per hill to reach approximately 83,000 plants ha $^{-1}$.

During seedbed preparation, phosphorus (P) at a rate of $120 \mathrm{~kg} \mathrm{P}_{2} \mathrm{O}_{5} \mathrm{ha}^{-1}$ as calcium superphosphate $\left(15.5 \% \mathrm{P}_{2} \mathrm{O}_{5}\right)$ was applied to the soil. Nitrogen (N) was applied at $240 \mathrm{~kg}$ $\mathrm{N} \mathrm{ha}{ }^{-1}$ as ammonium nitrate $(33.5 \% \mathrm{~N})$ in three equal doses; the first dose was applied directly after thinning, and the second and third doses were added immediately before the second and third irrigation, respectively. To apply $\mathrm{K}$ that was chosen as an experimental factor, it was applied at three levels $\left(\mathrm{K}_{1}=60, \mathrm{~K}_{2}=120\right.$, and $\mathrm{K}_{3}=180 \mathrm{~kg} \mathrm{~K}_{2} \mathrm{O}$ ha ${ }^{-1}$ ) as potassium sulfate $\left(48 \% \mathrm{~K}_{2} \mathrm{O}\right)$. To equalize $\mathrm{S}$ amount for all treatments related to $\mathrm{K}$ treatments, elemental $S$ was used for this purpose, as well as regarding the $S$ treatments using $\mathrm{CaSO}_{4}$. The $\mathrm{K}$ levels were arranged in subplots, and each $\mathrm{K}$ level was divided into two equal doses - the first dose was applied upon sowing and the second dose after thinning. To apply sulfur $(S)$ that was chosen as another experimental factor, it was applied at three levels $\left(S_{1}=175, S_{2}=350\right.$, and $\left.S_{3}=525 \mathrm{~kg} \mathrm{CaSO}_{4} \mathrm{ha}^{-1}\right)$ as calcium polysulfide $\left(\mathrm{CaSO}_{4}, 30 \% \mathrm{~S}\right)$. The $\mathrm{S}$ levels were arranged in sub-sub plots, and each $\mathrm{S}$ level was divided into two equal doses applied at 45 and 70 DAS. Thus, nine treatments were maintained (i.e., $K_{1} S_{1}, K_{1} S_{2}, K_{1} S_{3}, K_{2} S_{1}, K_{2} S_{2}, K_{2} S_{3}, K_{3} S_{1}, K_{3} S_{2}, K_{3} S_{3}$ ) on two sowing dates (early and late sowing) in two years. 
Table 1. Pre-season physicochemical characteristics of the tested soil (0-50 cm depth) for the 2018/19 and 2019/20 seasons.

\begin{tabular}{|c|c|c|}
\hline Characteristics & 2018/19 & $2019 / 20$ \\
\hline \multicolumn{3}{|c|}{ Particle size analysis } \\
\hline Sand $(\%)$ & 73.10 & 81.98 \\
\hline Silt (\%) & 13.47 & 9.07 \\
\hline Clay $(\%)$ & 13.43 & 8.95 \\
\hline Soil textural class & \multicolumn{2}{|c|}{ Sandy loam } \\
\hline \multicolumn{3}{|c|}{ Physical and chemical analysis } \\
\hline $\mathrm{pH} *$ & 7.55 & 8.35 \\
\hline $\mathrm{ECe}^{* *}\left(\mathrm{dS} \mathrm{m}^{-1}\right)$ & 8.56 & 9.36 \\
\hline $\mathrm{CaCO}_{3}(\%)$ & 7.83 & 8.03 \\
\hline Organic matter $(\%)$ & 1.11 & 0.85 \\
\hline \multicolumn{3}{|c|}{ Soluble cations ${ }^{* *}\left(\mathrm{mmolc} \mathrm{L}^{-1}\right)$} \\
\hline $\mathrm{Ca}^{2+}$ & 22.84 & 20.80 \\
\hline $\mathrm{K}^{+}$ & 7.594 & 3.594 \\
\hline \multicolumn{3}{|c|}{ Soluble anions ${ }^{* *}\left(\right.$ mmolc L $\left.^{-1}\right)$} \\
\hline $\mathrm{HCO}_{3}{ }^{-}$ & 8.416 & 10.416 \\
\hline $\mathrm{SO}_{4}^{2-}$ & 22.11 & 24.01 \\
\hline \multicolumn{3}{|c|}{ Available nutrients ( $\mathrm{mg} \mathrm{kg}^{-1}$ soil) } \\
\hline Nitrogen $(\mathrm{N})$ & 0.07 & 0.01 \\
\hline Phosphorus (P) & 3.84 & 3.24 \\
\hline Potassium $(\mathrm{K})$ & 42.2 & 36.0 \\
\hline Zinc $(\mathrm{Zn})$ & 1.80 & 1.30 \\
\hline Manganese (Mn) & 5.91 & 3.91 \\
\hline Iron $(\mathrm{Fe})$ & 0.88 & 0.56 \\
\hline Boron (B) & 0.46 & 0.20 \\
\hline
\end{tabular}

*Suspension of soil: $\mathrm{H}_{2} \mathrm{O}(1: 1, w / v)$ and ${ }^{* *}$ Soil paste extract 1:2.5 soil: $\mathrm{H}_{2} \mathrm{O}(w / v)$ for soil characteristics.

\subsection{Environmental Growing Conditions}

Table 2 shows the values of thermal units during the trial period in two growing seasons (obtained from the Fayoum meteorological station). The number of days was computed from the date of sowing to the date of harvest. Growing degree days (GDD) were computed by total daily mean values of temperatures minus the temperature base value of $3{ }^{\circ} \mathrm{C}[39$ ]. GDD values were computed for sugar beet using the following equation:

$\mathrm{GDD}=[($ Maximum temperature + Minimum temperature $) \div 2]-$ Temperature base $\left(3^{\circ} \mathrm{C}\right)$

Table 2. The values of thermal units during the trial period in two growing seasons (obtained from the Fayoum meteorological station).

\begin{tabular}{lcclcc}
\hline Thermal Units & \multicolumn{7}{c}{ 2018/2019 } & \multirow{2}{c}{ 2019/2020 } \\
\hline \multirow{2}{*}{ Month } & Early & Late & & Early & Late \\
\cline { 2 - 5 } & 755.3 & - & September, 2019 & 750.8 & - \\
\cline { 2 - 3 } September, 2018 & 670.1 & 670.1 & October, 2019 & 701.1 & 701.1 \\
October, 2018 & 503.0 & 503.0 & November, 2019 & 548.8 & 548.8 \\
November, 2018 & 349.2 & 349.2 & December, 2019 & 361.1 & 361.1 \\
December, 2018 & 276.4 & 276.4 & January, 2020 & 278.7 & 278.7 \\
January, 2019 & 305.9 & 305.9 & February, 2020 & 314.2 & 314.2 \\
February, 2019 & 414.5 & 414.5 & March, 2020 & 438.2 & 438.2 \\
March, 2019 & - & 528.5 & April, 2020 & - & 533.6 \\
April, 2019 & 3274.3 & 3047.5 & & 3392.8 & 3175.6 \\
Total & & & & & \\
\hline
\end{tabular}


All other cultural practices for the cultivation of sugar beet such as weed control and irrigation were carried out as recommended by the Egyptian Ministry of Agriculture and Land Reclamation.

\subsection{Sampling}

For all determinations, sampling was done twice from all sub-sub plots in the two seasons. At 90 days after sowing (DAS), the first sample was taken to assess vegetative growth traits according to the sowing date. The sample was composed of five fullyexpanded upper leaves taken from four sugar beet plants randomly selected to measure both the chlorophyll concentration (SPAD values) and the chlorophyll fluorescence (Fv/Fm) as physiological parameters. The second sample was taken at 210 DAS (harvest stage) from all sub-sub plots in both seasons to evaluate the yield and quality traits according to the sowing date. Each sample consisted of six randomly selected plants, which they were completely removed after irrigation of the soil to facilitate obtaining the plant with the whole root. The plants were then cleaned with tap water and separated into roots and tops to estimate their morphological characteristics. Sugar beet plants from all rows were then collected in each sub-sub plot, plus the six previously sampled plants that were all used to measure yield traits.

\subsection{Morpho-Physiological and Yield Attributes}

At 90 DAS, the selected samples were subjected to measure SPAD values (the chlorophyll concentration) using a chlorophyll meter (SPAD-502, Plus Konica Minolta, Inc., Tokyo, Japan). To obtain accurate SPAD values, each measurement was performed on both the second and third leaves and the mean of the two readings was recorded for each replicate. The Fv/Fm (chlorophyll fluorescence) was recorded by using (Handy PEA, Hansatech Instruments Ltd., Kings Lynn, UK), as described in [40], while the PI (performance index) was measured as described in [41]. At harvest (210 DAS) in both seasons, the sampled plants were separated into roots and tops to estimate the following traits: root length and diameter $(\mathrm{cm})$ were measured using a meter scale, while root fresh weight and top fresh weight $\left(\mathrm{kg} \mathrm{plant}^{-1}\right)$ were measured using a digital balance. Leaf area index (LAI) was measured using the following equation [42]:

$$
\text { LAI }=\text { Leaf area per plant }\left(\mathrm{cm}^{2}\right) \div \text { Plant ground area }\left(\mathrm{cm}^{2}\right)
$$

where leaf area per plant was measured using a leaf disc method [42], and plant ground area was assessed by multiplying the distance among plants $(20 \mathrm{~cm})$ by row width $(60 \mathrm{~cm})$.

\subsubsection{Juice Quality Traits}

Gross sugar content (\%) was determined in [43]. Pure sugar content (\%) was calculated according to [44]. The content of impurities in terms sodium (Na), potassium (K), and $\alpha$-amino-N in (meq per $100 \mathrm{~g}$ root) were determined by an Automatic Sugar Polarimetric. Loss sugar (\%), purity (\%), and alkalinity index were calculated by the following equations:

$$
\begin{gathered}
\text { Loss sugar }(\%)=\text { gross sugar }(\%)-\text { pure sugar }(\%), \\
\text { Purity }(\%)=[\text { Pure sugar }(\%) \div \text { Gross sugar }(\%)] \times 100, \\
\text { Alkalinity index }=(\mathrm{K}+\mathrm{Na}) \div \alpha \text {-amino } \mathrm{N}
\end{gathered}
$$

Impurity index was computed from $\mathrm{Na}, \mathrm{K}$, and $\alpha$-amino- $\mathrm{N}$ values and gross sugar by the following formula (and with absolute values):

$$
\text { Impurity index }=(10 \times \alpha \text {-amino } \mathrm{N}+3.5 \times \mathrm{Na}+2.5 \times \mathrm{K}) \div \text { Gross sugar }(\%)
$$




\subsubsection{Yield Traits}

Sugar beet plants from all rows of each sub-sub-plot were weighed, in addition to weighing six plants that were previously sampled and then converted to root yield $\left(\mathrm{Mg} \mathrm{ha}^{-1}\right)$ and top yield $\left(\mathrm{Mg} \mathrm{ha}^{-1}\right)$, along with biological yield $\left(\mathrm{Mg} \mathrm{ha}^{-1}\right)$, which was computed by adding the root yield to the top yield $\left(\mathrm{Mg} \mathrm{ha}^{-1}\right)$. Gross sugar yield $\left(\mathrm{Mg} \mathrm{ha}^{-1}\right)$ was calculated by multiplying the root yield by the gross sugar (\%). Pure sugar yield (Mg $\mathrm{ha}^{-1}$ ) was computed by multiplying the root yield by the pure sugar (\%). Harvest index (HI) was computed as follows:

$$
\mathrm{HI}=\left[\operatorname{Root} \text { yield }\left(\mathrm{Mg} \mathrm{ha}^{-1}\right)\right] \div\left[\operatorname{Root} \text { yield }\left(\mathrm{Mg} \mathrm{ha}^{-1}\right)+\text { Top yield }\left(\mathrm{Mg} \mathrm{ha}^{-1}\right)\right.
$$

The K-use efficiency was computed based on root yield (R-KUE as kg root per kg K) and the S-use efficiency was also computed based on root yield (R-SUE as $\mathrm{kg}$ root per $\mathrm{kg} \mathrm{S}$ ) by dividing the root yield using $\mathrm{K}$ and $\mathrm{S}$ rates, respectively.

\subsection{Statistical Analysis}

The data obtained were statistically analyzed by the technique of analysis of variance (ANOVA) for the split-split plot arranged in randomized complete blocks design using MSTAT-C (MI, USA). Fixed factors were sowing dates, potassium, and sulfur fertilization, while replications were the random factor. Duncan's Multiple Range Test was practiced at $5 \%$ and $1 \%$ levels of probability to test the differences between treatment means. Correlations and regressions were implemented by IBM SSPS Statistical 21st ed.

\section{Results}

The use of the highest levels of both potassium (K) fertilizer and sulfur (S) fertilizer combined with early sowing on the 1st of September provided temperatures and nutrition suitable for the growth of sugar beet plants to overcome the conditions of salinity in soil located in a semi-arid environment and secure adequate yields of high quality.

\subsection{Effect of Sowing Dates on Sugar Beet Physiological, Growth and Yield Traits}

The data listed in Tables 3-5 display that sowing sugar beet early (September 1st; $\left.\mathrm{D}_{1}\right)$ significantly $(p \leq 0.05)$ positively affected $\mathrm{Fv} / \mathrm{Fm}$ (chlorophyll fluorescence) and PI (performance index) in the 2018/2019 season, SPAD (chlorophyll concentration) in the $2019 / 2020$ season, while PI was highly affected $(p \leq 0.01)$ in the $2019 / 2020$ season by sowing early $\left(D_{1}\right)$. There are significant $(p \leq 0.05$ and 0.01$)$ variations between the two sowing dates $\left(\mathrm{D}_{1}\right.$ : September $1 \mathrm{st}$; and $\mathrm{D}_{2}$ : October $\left.1 \mathrm{st}\right)$ for root length and diameter, and root and top fresh weight plant ${ }^{-1}$ in both seasons (2018/2019 and 2019/2020), along with leaf area index (LAI) in the second (2019/20) season. There are significant $(p \leq 0.05)$ variations between the two sowing dates (D) for impurity index, loss sugar (\%), and purity (\%) in both seasons (2018/2019 and 2019/2020), $\alpha$-amino $\mathrm{N}$ and alkalinity index in the 1st season, and pure sugar (\%) in the second season. The data in Tables 5 and 6 reveal that the two sowing dates (D) showed significant $(p \leq 0.01)$ variations in yields (e.g., top, biological, gross, and pure sugar) of sugar beet plants in two seasons (2018/2019 and 2019/2020), as well as in root yield, R-KUE, and R-SUE in the second season. Harvest index significantly $(p \leq 0.05)$ varied in both seasons, and root yield, R-KUE, and R-SUE significantly $(p \leq 0.05)$ varied in the first season. $\mathrm{D}_{1}$ significantly outperformed late sowing (October 1st; $\mathrm{D}_{2}$ ) by 2.44\% (in the 2018/2019) for Fv /Fm, 50.84\% (in the 2018/2019) and $57.44 \%$ (in the 2019/2020) for PI, and $11.47 \%$ (in the 2019/2020) for SPAD. Additionally, $\mathrm{D}_{1}$ significantly outperformed $\mathrm{D}_{2}$ for root length by $13.99 \%$ and $14.70 \%$, root diameter by $16.34 \%$ and $14.77 \%$, root fresh weight by $21.75 \%$ and $15.36 \%$, and top fresh weight by $15.36 \%$, and $26.92 \%$ in the first and second seasons, respectively, along with LAI by $16.96 \%$ in the second season. The highest pure sugar content $(18.80 \%$ in the second season), purity $(90.0 \%$ and $89.5 \%$ in the first and second seasons, respectively), and alkalinity index (4.60 in the first season) were detected with early sown plants $\left(D_{1}\right)$, while the highest 
$\alpha$-amino $\mathrm{N}$ (1.64 in the first season), impurity index (1.64 and 1.68 in the first and second seasons, respectively), and loss sugar $(2.39 \%$ and $2.52 \%$ in the first and second seasons, respectively) were detected with late sowing $\left(D_{2}\right)$. Early sowing $\left(D_{1}\right)$ significantly increased root, top, biological, gross, and pure sugar yields, and R-KUE and R-SUE (83.6, 30.0, 113.88, $17.73,15.99 \mathrm{Mg} \mathrm{ha}^{-1}, 0.87 \mathrm{~kg}$ root per $\mathrm{kg} \mathrm{K}$, and $0.28 \mathrm{~kg}$ root per $\mathrm{kg} \mathrm{S}$, respectively, in the first season, and 82.6, 32.2, 114.84, 17.42, $15.61 \mathrm{Mg} \mathrm{ha}^{-1}, 1.45 \mathrm{~kg}$ root per $\mathrm{kg} \mathrm{K}$, and $0.28 \mathrm{~kg}$ root per $\mathrm{kg} \mathrm{S}$, respectively, in the second season). On the contrary, the lowest values $(0.73$ and 0.73 , respectively) of the harvest index and aforementioned yield traits were produced from late sowing $\left(\mathrm{D}_{2}\right)$ in both two seasons.

Table 3. Effect of sowing dates and applications of potassium and sulfur level and their interactions on photosynthetic efficiency (Fv / Fm, PI (\%), and SPAD values), root length $(\mathrm{cm})$, root diameter $(\mathrm{cm})$, and root fresh weight $\left(\mathrm{g}\right.$ plant $\left.{ }^{-1}\right)$ of sugar beet during two growing seasons (Mean $\pm \mathrm{SE}$ ) under soil salinity $\left(\mathrm{ECe}=8.96 \mathrm{dS} \mathrm{m}^{-1}\right)$ conditions.

\begin{tabular}{|c|c|c|c|c|c|c|}
\hline \multirow{2}{*}{ Treatments } & \multicolumn{2}{|c|}{ Fv/Fm } & \multicolumn{2}{|c|}{ PI (\%) } & \multicolumn{2}{|c|}{ SPAD Values } \\
\hline & $2018 / 2019$ & $2019 / 2020$ & $2018 / 2019$ & $2019 / 2020$ & $2018 / 2019$ & $2019 / 2020$ \\
\hline & \multicolumn{6}{|c|}{ Sowing dates (D) } \\
\hline $\mathrm{D}_{1}$ & $0.84 \pm 0.002 \mathrm{a}$ & $0.84 \pm 0.003 a$ & $8.04 \pm 0.23 \mathrm{a}$ & $8.25 \pm 0.25 a$ & $55.17 \pm 1.17 \mathrm{a}$ & $56.37 \pm 1.01 \mathrm{a}$ \\
\hline $\mathrm{D}_{2}$ & $0.82 \pm 0.002 b$ & $0.83 \pm 0.002 \mathrm{a}$ & $5.33 \pm 0.17 b$ & $5.24 \pm 0.17 b$ & $49.93 \pm 0.47 a$ & $50.57 \pm 0.55 b$ \\
\hline \multirow[t]{2}{*}{$p$-value } & $0.038 *$ & $0.127^{\mathrm{ns}}$ & $0.015^{*}$ & $0.005^{* *}$ & $0.088^{\mathrm{ns}}$ & $0.03 *$ \\
\hline & \multicolumn{6}{|c|}{ Potassium (K) application } \\
\hline $\mathrm{K}_{1}$ & $0.81 \pm 0.003 \mathrm{c}$ & $0.82 \pm 0.002 b$ & $5.02 \pm 0.47 c$ & $4.93 \pm 0.37 c$ & $48.31 \pm 1.45 c$ & $49.96 \pm 0.88 c$ \\
\hline $\mathrm{K}_{2}$ & $0.83 \pm 0.002 b$ & $0.83 \pm 0.002 b$ & $6.59 \pm 0.35 b$ & $6.80 \pm 0.42 b$ & $52.58 \pm 1.19 b$ & $52.77 \pm 0.99 b$ \\
\hline $\mathrm{K}_{3}$ & $0.85 \pm 0.001 \mathrm{a}$ & $0.85 \pm 0.003 a$ & $8.44 \pm 0.29 a$ & $8.50 \pm 0.51 a$ & $56.76 \pm 0.84 a$ & $57.67 \pm 1.29 \mathrm{a}$ \\
\hline \multirow[t]{2}{*}{$p$-value } & $<0.001^{* *}$ & $<0.001^{* *}$ & $<0.001^{* *}$ & $<0.001^{* *}$ & $<0.001^{* *}$ & $<0.001^{* *}$ \\
\hline & \multicolumn{6}{|c|}{ Sulphur (S) application } \\
\hline $\mathrm{S}_{1}$ & $0.82 \pm 0.003 c$ & $0.83 \pm 0.003 a$ & $6.08 \pm 0.43 c$ & $6.21 \pm 0.50 c$ & $49.53 \pm 0.99 c$ & $50.78 \pm 1.17 c$ \\
\hline $\mathrm{S}_{2}$ & $0.83 \pm 0.003 b$ & $0.84 \pm 0.004 \mathrm{a}$ & $6.68 \pm 0.49 b$ & $6.69 \pm 0.56 b$ & $52.94 \pm 1.38 b$ & $52.81 \pm 1.49 b$ \\
\hline $\mathrm{S}_{3}$ & $0.84 \pm 0.004 \mathrm{a}$ & $0.84 \pm 0.005 \mathrm{a}$ & $7.30 \pm 0.54 a$ & $7.34 \pm 0.45 a$ & $55.17 \pm 1.55 \mathrm{a}$ & $55.81 \pm 0.91 \mathrm{a}$ \\
\hline$p$-value & $<0.001^{* *}$ & $0.089 \mathrm{~ns}$ & $<0.001^{* *}$ & $<0.001^{* *}$ & $<0.001^{* *}$ & $<0.001^{* *}$ \\
\hline \multirow{2}{*}{ Treatments } & \multicolumn{2}{|c|}{ Root Length (cm) } & \multicolumn{2}{|c|}{ Root Diameter (cm) } & \multicolumn{2}{|c|}{ Root FW (g plant ${ }^{-1}$ ) } \\
\hline & $2018 / 2019$ & $2019 / 2020$ & $2018 / 2019$ & $2019 / 2020$ & $2018 / 2019$ & $2019 / 2020$ \\
\hline & \multicolumn{6}{|c|}{ Sowing dates (D) } \\
\hline $\mathrm{D}_{1}$ & $32.0 \pm 0.40 \mathrm{a}$ & $32.7 \pm 0.65 a$ & $13.6 \pm 0.15 a$ & $14.2 \pm 0.37 a$ & $1470 \pm 20.99 a$ & $1463 \pm 42.48 a$ \\
\hline $\mathrm{D}_{2}$ & $28.0 \pm 0.24 b$ & $28.5 \pm 0.31 b$ & $11.7 \pm 0.19 b$ & $12.4 \pm 0.15 b$ & $1208 \pm 22.29 b$ & $1268 \pm 18.48 b$ \\
\hline \multirow[t]{2}{*}{$p$-value } & 0.034 * & 0.032 * & $0.006^{* *}$ & 0.017 * & $0.019 *$ & $0.006^{* *}$ \\
\hline & \multicolumn{6}{|c|}{ Potassium $(\mathrm{K})$ application } \\
\hline $\mathrm{K}_{1}$ & $27.6 \pm 0.97 c$ & $28.4 \pm 0.49 c$ & $11.7 \pm 0.59 b$ & $12.5 \pm 0.19 c$ & $1239 \pm 1.01 b$ & $1269 \pm 22.50 c$ \\
\hline $\mathrm{K}_{2}$ & $29.7 \pm 0.51 b$ & $30.2 \pm 0.48 b$ & $12.3 \pm 0.27 b$ & $12.9 \pm 0.16 b$ & $1296 \pm 35.92 b$ & $1322 \pm 19.80 b$ \\
\hline $\mathrm{K}_{3}$ & $32.6 \pm 0.45 a$ & $33.3 \pm 0.82 a$ & $13.9 \pm 0.25 a$ & $14.5 \pm 0.39 a$ & $1482 \pm 32.18 \mathrm{a}$ & $1507 \pm 43.20 \mathrm{a}$ \\
\hline \multirow[t]{2}{*}{$p$-value } & $<0.001^{* *}$ & $<0.001^{* *}$ & $<0.001^{* *}$ & $<0.001^{* *}$ & $<0.001^{* *}$ & $<0.001^{* *}$ \\
\hline & \multicolumn{6}{|c|}{ Sulphur (S) application } \\
\hline $\mathrm{S}_{1}$ & $28.5 \pm 0.56 c$ & $29.5 \pm 0.61 c$ & $11.6 \pm 0.24 c$ & $12.4 \pm 0.28 c$ & $1231 \pm 37.48 c$ & $1262 \pm 28.19 c$ \\
\hline $\mathrm{S}_{2}$ & $29.8 \pm 0.68 b$ & $30.2 \pm 1.06 b$ & $12.5 \pm 0.30 \mathrm{~b}$ & $13.2 \pm 0.47 b$ & $1316 \pm 39.52 b$ & $1357 \pm 55.33 b$ \\
\hline $\mathrm{S}_{3}$ & $31.7 \pm 1.01 \mathrm{a}$ & $32.2 \pm 0.60 \mathrm{a}$ & $13.9 \pm 0.58 a$ & $14.3 \pm 0.26 a$ & $1470+67.04 a$ & $1479 \pm 27.04 a$ \\
\hline$p$-value & $<0.001^{* *}$ & $<0.001^{* *}$ & $<0.001^{* *}$ & $<0.001^{* *}$ & $<0.001^{* *}$ & $<0.001^{* *}$ \\
\hline
\end{tabular}

$*{ }^{* *}$ indicate the significant variation at $(p \leq 0.05)$ and $(p \leq 0.01)$, respectively, and "ns" point to non-significant variation. Means sharing the same letter in each column are not significantly different. $\mathrm{D}_{1}=$ sowing at the 1 st of September; $\mathrm{D}_{2}=$ sowing at the 1 st of October; $\mathrm{K}_{1}=60 \mathrm{~kg} \mathrm{~K}_{2} \mathrm{O} \mathrm{ha}^{-1} ; \mathrm{K}_{2}=120 \mathrm{~kg} \mathrm{~K}_{2} \mathrm{O} \mathrm{ha}^{-1} ; \mathrm{K}_{3}=180 \mathrm{~kg} \mathrm{~K}_{2} \mathrm{O} \mathrm{ha}^{-1} ; \mathrm{S}_{1}=175 \mathrm{~kg} \mathrm{CaSO}_{4} \mathrm{ha}^{-1} ; \mathrm{S}_{2}=350 \mathrm{~kg} \mathrm{CaSO}_{4} \mathrm{ha}^{-1} ; \mathrm{S}_{3}=525 \mathrm{~kg} \mathrm{CaSO}_{4}$ $\mathrm{ha}^{-1}$; and $\mathrm{FW}=$ fresh weight. 
Table 4. Effect of sowing dates and applications of potassium and sulfur level and their interactions on top fresh weight $\left(\mathrm{kg} \mathrm{plant}^{-1}\right)$, leaf area index (LAI), sucrose (\%), $\mathrm{Na}, \mathrm{K}$, and $\alpha$-amino $\mathrm{N}$ (meq per $100 \mathrm{~g}$ ) and impurity index of sugar beet during two growing seasons (Mean $\pm \mathrm{SE}$ ) under soil salinity $\left(\mathrm{ECe}=8.96 \mathrm{dS} \mathrm{m}^{-1}\right)$ conditions.

\begin{tabular}{|c|c|c|c|c|c|c|c|c|}
\hline \multirow{2}{*}{ Treat } & \multicolumn{2}{|c|}{ Top FW (kg Plant $\left.{ }^{-1}\right)$} & \multicolumn{2}{|c|}{ LAI } & \multicolumn{2}{|c|}{ Gross Sugar (\%) } & \multicolumn{2}{|c|}{$\mathrm{Na}^{+}($meq Per $100 \mathrm{~g})$} \\
\hline & $2018 / 2019$ & $2019 / 2020$ & $2018 / 2019$ & $2019 / 2020$ & $2018 / 2019$ & $2019 / 2020$ & $2018 / 2019$ & $2019 / 2020$ \\
\hline & \multicolumn{8}{|c|}{ Sowing dates (D) } \\
\hline $\mathrm{D}_{1}$ & $0.99 \pm 0.02 a$ & $1.05 \pm 0.05 a$ & $5.23 \pm 0.12 \mathrm{a}$ & $5.31 \pm 0.28 \mathrm{a}$ & $21.1 \pm 0.16 \mathrm{a}$ & $21.1 \pm 0.26 \mathrm{a}$ & $1.55 \pm 0.03 a$ & $1.74 \pm 0.05 a$ \\
\hline $\mathrm{D}_{2}$ & $0.78 \pm 0.01 b$ & $0.84 \pm 0.01 b$ & $4.57 \pm 0.11 \mathrm{a}$ & $4.54 \pm 0.14 b$ & $20.0 \pm 0.14 \mathrm{a}$ & $20.2 \pm 0.17 \mathrm{a}$ & $1.73 \pm 0.04 \mathrm{a}$ & $2.01 \pm 0.04 \mathrm{a}$ \\
\hline \multirow[t]{2}{*}{$p$-value } & $0.002 * *$ & $0.005^{* *}$ & $0.088^{\mathrm{ns}}$ & $0.037 *$ & $0.169^{\mathrm{ns}}$ & $0.055^{\mathrm{ns}}$ & $0.062^{\mathrm{ns}}$ & $0.08^{\mathrm{ns}}$ \\
\hline & \multicolumn{8}{|c|}{ Potassium (K) application } \\
\hline $\mathrm{K}_{1}$ & $0.78 \pm 0.06 c$ & $0.85 \pm 0.02 c$ & $4.26 \pm 0.37 b$ & $4.45 \pm 0.08 b$ & $19.5 \pm 0.26 c$ & $19.7 \pm 0.25 c$ & $1.89 \pm 0.06 \mathrm{a}$ & $2.20 \pm 0.03 a$ \\
\hline $\mathrm{K}_{2}$ & $0.87 \pm 0.03 b$ & $0.91 \pm 0.02 b$ & $4.58 \pm 0.15 b$ & $4.61 \pm 0.17 \mathrm{~b}$ & $20.4 \pm 0.17 \mathrm{~b}$ & $20.4 \pm 0.21 b$ & $1.68 \pm 0.04 b$ & $1.88 \pm 0.05 b$ \\
\hline $\mathrm{K}_{3}$ & $1.01 \pm 0.02 \mathrm{a}$ & $1.07 \pm 0.05 \mathrm{a}$ & $5.85+0.13 a$ & $5.71 \pm 0.27 \mathrm{a}$ & $21.8 \pm 0.19 a$ & $21.6 \pm 0.29 a$ & $1.36 \pm 0.05 c$ & $1.55 \pm 0.09 c$ \\
\hline \multirow[t]{2}{*}{$p$-value } & $<0.001^{* *}$ & $<0.001^{* *}$ & $<0.001^{* *}$ & $<0.001^{* *}$ & $<0.001^{* *}$ & $<0.001^{* *}$ & $<0.001^{* *}$ & $<0.001^{* *}$ \\
\hline & \multicolumn{8}{|c|}{ Sulphur (S) application } \\
\hline $\mathrm{S}_{1}$ & $0.78 \pm 0.02 c$ & $0.83 \pm 0.03 c$ & $4.18 \pm 0.14 c$ & $4.20 \pm 0.19 c$ & $20.1 \pm 0.27 c$ & $20.1 \pm 0.25 c$ & $1.81 \pm 0.06 \mathrm{a}$ & $2.04 \pm 0.07 a$ \\
\hline $\mathrm{S}_{2}$ & $0.87 \pm 0.03 b$ & $0.93 \pm 0.06 b$ & $4.75 \pm 0.22 b$ & $4.74 \pm 0.29 b$ & $21.0 \pm 0.30 \mathrm{a}$ & $21.0 \pm 0.36 a$ & $1.67 \pm 0.05 b$ & $1.89 \pm 0.09 b$ \\
\hline \multirow{5}{*}{$\begin{array}{c}\mathrm{S}_{3} \\
p \text {-value }\end{array}$} & $1.01 \pm 0.06 \mathrm{a}$ & $1.07 \pm 0.02 \mathrm{a}$ & $5.76 \pm 0.35 a$ & $5.83 \pm 0.14 a$ & $20.6 \pm 0.30 b$ & $20.7 \pm 0.26 b$ & $1.45 \pm 0.07 c$ & $1.70 \pm 0.07 c$ \\
\hline & $<0.001^{* *}$ & $<0.001^{* *}$ & $<0.001^{* *}$ & $<0.001^{* *}$ & $<0.001^{* *}$ & $<0.001^{* *}$ & $<0.001^{* *}$ & $<0.001 * *$ \\
\hline & \multicolumn{2}{|c|}{$\mathrm{K}^{+}($meq Per $100 \mathrm{~g})$} & \multicolumn{2}{|c|}{$\begin{array}{c}\alpha \text {-Amino N } \\
(\mathrm{meq} \text { Per } 100 \mathrm{~g})\end{array}$} & \multicolumn{2}{|c|}{ Impurity Index } & & \\
\hline & $2018 / 2019$ & $2019 / 2020$ & $2018 / 2019$ & $2019 / 2020$ & $2018 / 2019$ & $2019 / 2020$ & & \\
\hline & \multicolumn{8}{|c|}{ Sowing dates (D) } \\
\hline $\mathrm{D}_{1}$ & $3.45 \pm 0.12 \mathrm{a}$ & $3.46 \pm 0.10 \mathrm{a}$ & $1.18 \pm 0.07 b$ & $1.29 \pm 0.05 a$ & $1.24 \pm 0.05 b$ & $1.33 \pm 0.05 b$ & & \\
\hline $\mathrm{D}_{2}$ & $4.04 \pm 0.15 a$ & $4.05 \pm 0.11 \mathrm{a}$ & $1.64 \pm 0.06 \mathrm{a}$ & $1.63 \pm 0.06 a$ & $1.64 \pm 0.05 a$ & $1.68 \pm 0.05 a$ & & \\
\hline \multirow[t]{2}{*}{$p$-value } & $0.065^{\mathrm{ns}}$ & $0.076^{\mathrm{ns}}$ & $0.039 *$ & $0.120^{\mathrm{ns}}$ & $0.003^{* *}$ & $0.011 *$ & & \\
\hline & \multicolumn{8}{|c|}{ Potassium (K) application } \\
\hline $\mathrm{K}_{1}$ & $4.51 \pm 0.12 \mathrm{a}$ & $4.49 \pm 0.11 \mathrm{a}$ & $1.80 \pm 0.09 a$ & $1.96 \pm 0.07 a$ & $1.85 \pm 0.06 a$ & $1.96 \pm 0.05 a$ & & \\
\hline $\mathrm{K}_{2}$ & $3.53 \pm 0.15 b$ & $3.54 \pm 0.14 b$ & $1.35 \pm 0.09 b$ & $1.41 \pm 0.06 b$ & $1.39 \pm 0.06 b$ & $1.45 \pm 0.05 b$ & & \\
\hline $\mathrm{K}_{3}$ & $3.21 \pm 0.16 c$ & $3.24 \pm 0.19 c$ & $1.07 \pm 0.07 \mathrm{c}$ & $1.01 \pm 0.11 c$ & $1.08 \pm 0.06 c$ & $1.10 \pm 0.10 c$ & & \\
\hline \multirow[t]{2}{*}{$p$-value } & $<0.001 * *$ & $<0.001 * *$ & $<0.001 * *$ & $<0.001^{* *}$ & $<0.001^{* *}$ & $<0.001 * *$ & & \\
\hline & \multicolumn{8}{|c|}{ Sulphur (S) application } \\
\hline $\mathrm{S}_{1}$ & $4.21 \pm 0.16 a$ & $4.22 \pm 0.15 a$ & $1.57 \pm 0.10 \mathrm{a}$ & $1.62 \pm 0.09 a$ & $1.53 \pm 0.09 a$ & $1.66 \pm 0.09 a$ & & \\
\hline $\mathrm{S}_{2}$ & $3.70 \pm 0.20 b$ & $3.66 \pm 0.17 b$ & $1.41 \pm 0.11 b$ & $1.45 \pm 0.12 b$ & $1.41 \pm 0.10 \mathrm{~b}$ & $1.46 \pm 0.11 b$ & & \\
\hline $\mathrm{S}_{3}$ & $3.34 \pm 0.16 c$ & $3.38 \pm 0.18 c$ & $1.24 \pm 0.10 \mathrm{c}$ & $1.32 \pm 0.10 \mathrm{c}$ & $1.38 \pm 0.09 c$ & $1.39 \pm 0.09 c$ & & \\
\hline$p$-value & $<0.001^{* *}$ & $<0.001^{* *}$ & $<0.001 * *$ & $<0.001 * *$ & $<0.001$ & $<0.001^{* *}$ & & \\
\hline
\end{tabular}

$*$ ** indicate the significant variation at $(p \leq 0.05)$ and $(p \leq 0.01)$, respectively, and "ns" point to non-significant variation. Means sharing the same letter in each column are not significantly different. $\mathrm{D}_{1}=$ sowing at the 1 st of September; $\mathrm{D}_{2}=$ sowing at the 1 st of October; $\mathrm{K}_{1}=60 \mathrm{~kg} \mathrm{~K}_{2} \mathrm{O} \mathrm{ha}{ }^{-1} ; \mathrm{K}_{2}=120 \mathrm{~kg} \mathrm{~K}_{2} \mathrm{O} \mathrm{ha}^{-1} ; \mathrm{K}_{3}=180 \mathrm{~kg} \mathrm{~K}_{2} \mathrm{O} \mathrm{ha}^{-1} ; \mathrm{S}_{1}=175 \mathrm{~kg} \mathrm{CaSO}_{4} \mathrm{ha}^{-1} ; \mathrm{S}_{2}=350 \mathrm{~kg} \mathrm{CaSO}_{4} \mathrm{ha}^{-1} ; \mathrm{S}_{3}=525 \mathrm{~kg} \mathrm{CaSO}_{4}$ $\mathrm{ha}^{-1}$; and $\mathrm{FW}=$ fresh weight. 
Table 5. Effect of sowing dates and applications of potassium and sulfur level and their interactions on pure sugar (\%), loss sugar (\%), purity (\%), alkalinity index, and yields of roots and tops $\left(\mathrm{Mg} \mathrm{ha}^{-1}\right)$ of sugar beet during two growing seasons $($ Mean $\pm \mathrm{SE})$ under soil salinity $\left(\mathrm{ECe}=8.96 \mathrm{dS} \mathrm{m}^{-1}\right)$ conditions.

\begin{tabular}{|c|c|c|c|c|c|c|}
\hline \multirow{2}{*}{ Treatments } & \multicolumn{2}{|c|}{ Pure Sugar (\%) } & \multicolumn{2}{|c|}{ Loss Sugar (\%) } & \multicolumn{2}{|c|}{ Purity (\%) } \\
\hline & $2018 / 2019$ & $2019 / 2020$ & $2018 / 2019$ & $2019 / 2020$ & $2018 / 2019$ & $2019 / 2020$ \\
\hline & \multicolumn{6}{|c|}{ Sowing dates (D) } \\
\hline $\mathrm{D}_{1}$ & $19.0 \pm 0.19 a$ & $18.8 \pm 0.25 a$ & $2.10 \pm 0.05 b$ & $2.20 \pm 0.04 b$ & $90.0 \pm 0.28 a$ & $89.5 \pm 0.21 a$ \\
\hline $\mathrm{D}_{2}$ & $17.6 \pm 0.16 a$ & $17.6 \pm 0.17 b$ & $2.39 \pm 0.05 a$ & $2.52 \pm 0.05 a$ & $88.0 \pm 0.29 b$ & $87.4 \pm 0.26 b$ \\
\hline \multirow[t]{2}{*}{$p$-value } & $0.111^{\text {ns }}$ & 0.026 * & 0.034 * & $0.041 *$ & $0.021 *$ & $0.021 *$ \\
\hline & \multicolumn{6}{|c|}{ Potassium (K) application } \\
\hline $\mathrm{K}_{1}$ & $16.9 \pm 0.27 c$ & $16.9 \pm 0.24 c$ & $2.60 \pm 0.04 a$ & $2.77 \pm 0.05 a$ & $86.6 \pm 0.22 c$ & $85.9 \pm 0.23 c$ \\
\hline $\mathrm{K}_{2}$ & $18.2 \pm 0.20 \mathrm{~b}$ & $18.1 \pm 0.22 b$ & $2.19 \pm 0.05 b$ & $2.28 \pm 0.06 b$ & $89.3 \pm 0.31 b$ & $88.8 \pm 0.33 b$ \\
\hline $\mathrm{K}_{3}$ & $19.9 \pm 0.21 \mathrm{a}$ & $19.6 \pm 0.34 a$ & $1.95 \pm 0.058 \mathrm{c}$ & $2.03 \pm 0.10 c$ & $91.0 \pm 0.31 \mathrm{a}$ & $90.6 \pm 0.58 a$ \\
\hline \multirow[t]{2}{*}{$p$-value } & $<0.001^{* *}$ & $<0.001^{* *}$ & $<0.001^{* *}$ & $<0.001^{* *}$ & $<0.001 * *$ & $<0.001^{* *}$ \\
\hline & \multicolumn{6}{|c|}{ Sulphur (S) application } \\
\hline$S_{1}$ & $17.9 \pm 0.33 c$ & $17.9 \pm 0.31 c$ & $2.33 \pm 0.07 a$ & $2.59 \pm 0.09 a$ & $88.6 \pm 0.50 b$ & $87.4 \pm 0.53 c$ \\
\hline $\mathrm{S}_{2}$ & $18.8 \pm 0.38 \mathrm{a}$ & $18.6 \pm 0.42 \mathrm{a}$ & $2.24+0.09 b$ & $2.33 \pm 0.09 b$ & $89.1 \pm 0.54 a$ & $88.8 \pm 0.61 b$ \\
\hline $\mathrm{S}_{3}$ & $18.3 \pm 0.36 b$ & $18.1 \pm 0.30 \mathrm{~b}$ & $2.17 \pm 0.07 c$ & $2.16 \pm 0.08 c$ & $89.3 \pm 0.50 \mathrm{a}$ & $89.2 \pm 0.48 a$ \\
\hline$p$-value & $<0.001^{* *}$ & $<0.001 * *$ & $<0.001 * *$ & $<0.001^{* *}$ & $<0.001^{* *}$ & $<0.001^{* *}$ \\
\hline \multirow{2}{*}{ Treatments } & \multicolumn{2}{|c|}{ Alkalinity Index } & \multicolumn{2}{|c|}{ Root Yield (Mg ha $\left.{ }^{-1}\right)$} & \multicolumn{2}{|c|}{ Top Yield (Mg ha $\left.{ }^{-1}\right)$} \\
\hline & $2018 / 2019$ & $2019 / 2020$ & $2018 / 2019$ & $2019 / 2020$ & $2018 / 2019$ & $2019 / 2020$ \\
\hline & \multicolumn{6}{|c|}{ Sowing dates (D) } \\
\hline $\mathrm{D}_{1}$ & $4.60 \pm 0.19 a$ & $4.28 \pm 0.16 a$ & $83.6 \pm 0.57 \mathrm{a}$ & $82.6 \pm 1.81 \mathrm{a}$ & $30.0 \pm 0.48 a$ & $32.2 \pm 0.55 a$ \\
\hline $\mathrm{D}_{2}$ & $3.67 \pm 0.11 b$ & $3.85 \pm 0.13 a$ & $69.8 \pm 1.05 b$ & $67.4 \pm 0.62 b$ & $18.9 \pm 0.43 b$ & $21.0 \pm 0.52 b$ \\
\hline \multirow[t]{2}{*}{$p$-value } & 0.018 * & $0.54^{\mathrm{ns}}$ & $0.02 *$ & $<0.001^{* *}$ & $0.004^{* *}$ & $0.001^{* *}$ \\
\hline & \multicolumn{6}{|c|}{ Potassium (K) application } \\
\hline $\mathrm{K}_{1}$ & $3.61 \pm 0.35 b$ & $3.43 \pm 0.17 c$ & $70.2 \pm 2.52 c$ & $69.5 \pm 1.62 c$ & $22.1 \pm 1.81 \mathrm{c}$ & $23.6 \pm 1.43 c$ \\
\hline $\mathrm{K}_{2}$ & $4.08 \pm 0.25 b$ & $3.93 \pm 0.16 b$ & $76.2 \pm 1.33 b$ & $73.8 \pm 1.60 \mathrm{~b}$ & $24.6 \pm 1.45 b$ & $26.4 \pm 1.33 b$ \\
\hline $\mathrm{K}_{3}$ & $4.72 \pm 0.13 a$ & $4.82 \pm 0.20 \mathrm{a}$ & $84.8 \pm 1.81 \mathrm{a}$ & $81.9 \pm 2.45 a$ & $27.2 \pm 1.26 a$ & $29.8 \pm 1.50 \mathrm{a}$ \\
\hline \multirow[t]{2}{*}{$p$-value } & $0.011 *$ & $<0.001^{* *}$ & $<0.001^{* *}$ & $<0.001^{* *}$ & $<0.001^{* *}$ & $<0.001^{* *}$ \\
\hline & \multicolumn{6}{|c|}{ Sulphur (S) application } \\
\hline$S_{1}$ & $3.39 \pm 0.12 c$ & $4.06 \pm 0.18 \mathrm{a}$ & $74.3 \pm 1.79 c$ & $71.9 \pm 1.98 c$ & $22.6 \pm 1.33 c$ & $24.8 \pm 1.48 c$ \\
\hline $\mathrm{S}_{2}$ & $4.05 \pm 0.22 b$ & $4.03 \pm 0.19 a$ & $76.4 \pm 2.00 \mathrm{~b}$ & $74.5 \pm 3.12 b$ & $24.5 \pm 1.50 \mathrm{~b}$ & $26.5 \pm 1.60 b$ \\
\hline $\mathrm{S}_{3}$ & $4.98 \pm 0.31 a$ & $4.10 \pm 0.17 \mathrm{a}$ & $80.5 \pm 3.02 \mathrm{a}$ & $78.8 \pm 1.74 a$ & $26.8 \pm 1.74 a$ & $28.5 \pm 1.38 a$ \\
\hline$p$-value & $<0.001^{* *}$ & $0.670^{\mathrm{ns}}$ & $<0.001^{* *}$ & $<0.001^{* *}$ & $<0.001^{* *}$ & $<0.001^{* *}$ \\
\hline
\end{tabular}

${ }^{*}{ }^{* *}$ indicate the significant variation at $(p \leq 0.05)$ and $(p \leq 0.01)$, respectively, and "ns" point to non-significant variation. Means sharing the same letter in each column are not significantly different. $\mathrm{D}_{1}=$ sowing at the 1st of September; $\mathrm{D}_{2}=$ sowing at the 1 st of October; $\mathrm{K}_{1}=60 \mathrm{~kg} \mathrm{~K}_{2} \mathrm{O} \mathrm{ha}{ }^{-1} ; \mathrm{K}_{2}=120 \mathrm{~kg} \mathrm{~K}_{2} \mathrm{O} \mathrm{ha}^{-1} ; \mathrm{K}_{3}=180 \mathrm{~kg} \mathrm{~K}_{2} \mathrm{O} \mathrm{ha}^{-1} ; \mathrm{S}_{1}=175 \mathrm{~kg} \mathrm{CaSO}_{4} \mathrm{ha}^{-1} ; \mathrm{S}_{2}=350 \mathrm{~kg} \mathrm{CaSO}_{4}$ ha $^{-1}$; and S $3=525 \mathrm{~kg}$ $\mathrm{CaSO}_{4} \mathrm{ha}^{-1}$. 
Table 6. Effect of sowing dates and applications of potassium and sulfur level and their interactions on harvest index, biological, gross sugar, and pure sugar yields $\left(\mathrm{Mg} \mathrm{ha}^{-1}\right)$, harvest index, and use efficiency of $\mathrm{K}$ and $\mathrm{S}$ of sugar beet during two growing seasons (Mean $\pm \mathrm{SE}$ ) under soil salinity $\left(\mathrm{ECe}=8.96 \mathrm{dS} \mathrm{m}^{-1}\right)$ conditions.

\begin{tabular}{|c|c|c|c|c|c|c|}
\hline \multirow{2}{*}{ Treatments } & \multicolumn{2}{|c|}{ Biological Yield (Mg ha ${ }^{-1}$ ) } & \multicolumn{2}{|c|}{ Harvest Index } & \multicolumn{2}{|c|}{ Gross Sugar Yield (Mg ha $\left.{ }^{-1}\right)$} \\
\hline & $2018 / 2019$ & $2019 / 2020$ & $2018 / 2019$ & $2019 / 2020$ & $2018 / 2019$ & $2019 / 2020$ \\
\hline & \multicolumn{6}{|c|}{ Sowing dates $(\mathrm{D})$} \\
\hline $\mathrm{D}_{1}$ & $113.88 \pm 0.42 \mathrm{a}$ & $114.84 \pm 0.9 \mathrm{a}$ & $0.73 \pm 0.01 b$ & $0.73 \pm 0.01 b$ & $17.73 \pm 0.08 \mathrm{a}$ & $17.42 \pm 0.25 \mathrm{a}$ \\
\hline $\mathrm{D}_{2}$ & $89.25 \pm 0.50 b$ & $88.49 \pm 0.43 b$ & $0.79 \pm 0.01 \mathrm{a}$ & $0.76 \pm 0.01 \mathrm{a}$ & $14.11 \pm 0.10 \mathrm{~b}$ & $13.61 \pm 0.08 b$ \\
\hline \multirow[t]{2}{*}{$p$-value } & $0.005^{* *}$ & $<0.001 * *$ & $0.039 *$ & $0.028 *$ & $0.006^{* *}$ & $0.005^{* *}$ \\
\hline & \multicolumn{6}{|c|}{ Potassium (K) application } \\
\hline $\mathrm{K}_{1}$ & $92.20 \pm 1.78 c$ & $93.15 \pm 1.26 c$ & $0.76 \pm 0.01 \mathrm{a}$ & $0.75 \pm 0.01 \mathrm{a}$ & $13.76 \pm 0.31 c$ & $13.69 \pm 0.016 c$ \\
\hline $\mathrm{K}_{2}$ & $100.72 \pm 1.11 b$ & $100.15 \pm 1.20 \mathrm{~b}$ & $0.76 \pm 0.01 a$ & $0.74 \pm 0.01 \mathrm{a}$ & $15.57 \pm 0.15 b$ & $15.07 \pm 0.15 b$ \\
\hline $\mathrm{K}_{3}$ & $111.76 \pm 1.26 \mathrm{a}$ & $111.65 \pm 1.62 \mathrm{a}$ & $0.76 \pm 0.01 \mathrm{a}$ & $0.74 \pm 0.01 \mathrm{a}$ & $18.52 \pm 0.18 \mathrm{a}$ & $17.83 \pm 0.30 \mathrm{a}$ \\
\hline \multirow[t]{2}{*}{$p$-value } & $<0.001 * *$ & $<0.001 * *$ & $0.318^{\mathrm{ns}}$ & $0.443^{\mathrm{ns}}$ & $<0.001 * *$ & $<0.001^{* *}$ \\
\hline & \multicolumn{6}{|c|}{ Sulphur (S) application } \\
\hline $\mathrm{S}_{1}$ & $96.72 \pm 1.25 c$ & $96.75 \pm 1.42 c$ & $0.77 \pm 0.01 \mathrm{a}$ & $075 \pm 0.01 \mathrm{a}$ & $14.95 \pm 0.22 c$ & $14.47 \pm 0.23 c$ \\
\hline $\mathrm{S}_{2}$ & $100.75 \pm 1.41 b$ & $100.96 \pm 1.94 b$ & $0.76 \pm 0.01 b$ & $0.74 \pm 0.01 \mathrm{a}$ & $16.14 \pm 0.26 b$ & $15.64 \pm 0.40 b$ \\
\hline $\mathrm{S}_{3}$ & $107.24 \pm 1.94 a$ & $107.27 \pm 1.28 \mathrm{a}$ & $0.75 \pm 0.01 \mathrm{c}$ & $0.75 \pm 0.01 \mathrm{a}$ & $16.71 \pm 0.37 a$ & $16.47 \pm 0.19 a$ \\
\hline$p$-value & $<0.001^{* *}$ & $<0.001 * *$ & $<0.001^{* *}$ & $0.069^{\mathrm{ns}}$ & $<0.001^{* *}$ & $<0.001 * *$ \\
\hline \multirow{2}{*}{ Treatments } & \multicolumn{2}{|c|}{ Pure Sugar Yield $\left(\mathrm{Mg} \mathrm{ha}^{-1}\right)$} & \multicolumn{2}{|c|}{ R-KUE (kg Root kg K ${ }^{-1}$ ) } & \multicolumn{2}{|c|}{ R-SUE (kg Root kg S-1) } \\
\hline & $2018 / 2019$ & $2019 / 2020$ & $2018 / 2019$ & $2019 / 2020$ & $2018 / 2019$ & $2019 / 2020$ \\
\hline & \multicolumn{6}{|c|}{ Sowing dates (D) } \\
\hline $\mathrm{D}_{1}$ & $15.99 \pm 0.09 a$ & $15.61 \pm 0.23 a$ & $0.87 \pm 0.06 a$ & $1.45 \pm 0.03 a$ & $0.28 \pm 0.02 \mathrm{a}$ & $0.28 \pm 0.02 \mathrm{a}$ \\
\hline $\mathrm{D}_{2}$ & $12.45 \pm 0.10 \mathrm{~b}$ & $11.92 \pm 0.08 b$ & $0.73 \pm 0.05 b$ & $1.18 \pm 0.01 b$ & $0.24 \pm 0.02 b$ & $0.23 \pm 0.02 b$ \\
\hline \multirow[t]{2}{*}{$p$-value } & $0.007^{* *}$ & $0.005^{* *}$ & $0.032 *$ & $0.005^{* *}$ & 0.03 * & $<0.001^{* *}$ \\
\hline & \multicolumn{6}{|c|}{ Potassium (K) application } \\
\hline $\mathrm{K}_{1}$ & $11.90 \pm 0.26 c$ & $11.75 \pm 0.15 c$ & $1.23 \pm 0.01 \mathrm{a}$ & $1.43 \pm 0.04 \mathrm{a}$ & $0.24 \pm 0.03 c$ & $0.24 \pm 0.01 c$ \\
\hline $\mathrm{K}_{2}$ & $13.90 \pm 0.15 b$ & $13.40 \pm 0.15 b$ & $0.67 \pm 0.01 b$ & $1.29 \pm 0.03 b$ & $0.26 \pm 0.03 b$ & $0.25 \pm 0.01 b$ \\
\hline $\mathrm{K}_{3}$ & $16.87 \pm 0.17 \mathrm{a}$ & $16.16 \pm 0.29 a$ & $0.49 \pm 0.03 c$ & $1.22 \pm 0.03 c$ & $0.28 \pm 0.03 a$ & $0.27 \pm 0.03 a$ \\
\hline \multirow[t]{2}{*}{$p$-value } & $<0.001^{* *}$ & $<0.001^{* *}$ & $<0.001^{* *}$ & $<0.001^{* *}$ & $<0.001^{* *}$ & $<0.001^{* *}$ \\
\hline & \multicolumn{6}{|c|}{ Sulphur (S) application } \\
\hline $\mathrm{S}_{1}$ & $13.35 \pm 0.22 c$ & $12.92 \pm 0.23 c$ & $0.77 \pm 0.08 \mathrm{c}$ & $1.26 \pm 0.03 b$ & $0.42 \pm 0.01 \mathrm{a}$ & $0.40 \pm 0.01 \mathrm{a}$ \\
\hline $\mathrm{S}_{2}$ & $14.45 \pm 0.27 b$ & $13.92 \pm 0.38 b$ & $0.79 \pm 0.08 b$ & $1.30 \pm 0.05 a$ & $0.21 \pm 0.01 b$ & $0.21 \pm 0.01 b$ \\
\hline $\mathrm{S}_{3}$ & $14.90 \pm 0.36 a$ & $14.47 \pm 0.20 \mathrm{a}$ & $0.82 \pm 0.08 \mathrm{a}$ & $1.38 \pm 0.03 a$ & $0.15 \pm 0.01 c$ & $0.15 \pm 0.01 c$ \\
\hline$p$-value & $<0.001^{* *}$ & $<0.001 * *$ & $<0.001 * *$ & $<0.001 * *$ & $<0.001 * *$ & $<0.001^{* *}$ \\
\hline
\end{tabular}

$*, * *$ indicate the significant variation at $(p \leq 0.05)$ and $(p \leq 0.01)$, respectively, and "ns" point to non-significant variation. Means sharing the same letter in each column are not significantly different. $\mathrm{D}_{1}=$ sowing at the 1st of September; $\mathrm{D}_{2}=$ sowing at the 1 st of October; $\mathrm{K}_{1}=60 \mathrm{~kg} \mathrm{~K}_{2} \mathrm{O} \mathrm{ha}{ }^{-1} ; \mathrm{K}_{2}=120 \mathrm{~kg} \mathrm{~K}_{2} \mathrm{O} \mathrm{ha}^{-1} ; \mathrm{K}_{3}=180 \mathrm{~kg} \mathrm{~K}_{2} \mathrm{O} \mathrm{ha}^{-1} ; \mathrm{S}_{1}=175 \mathrm{~kg} \mathrm{CaSO}_{4} \mathrm{ha}^{-1} ; \mathrm{S}_{2}=350 \mathrm{~kg} \mathrm{CaSO}_{4} \mathrm{ha}^{-1} ;$ and S $_{3}=525 \mathrm{~kg}$ $\mathrm{CaSO}_{4} \mathrm{ha}^{-1}$.

\subsection{Effect of Potassium Fertilization on Sugar Beet Physiological, Growth and Yield Traits}

Potassium $(\mathrm{K})$ levels had significant $(p \leq 0.01)$ variations for Fv/Fm, PI, SPAD, root length and diameter, root and top fresh weight plant ${ }^{-1}, \mathrm{LAI}$, and for all juice quality traits (impurity index, loss sugar content, purity percentage, $\alpha$-amino $\mathrm{N}$, alkalinity index, and pure sugar content) in both seasons under soil salinity conditions $\left(\mathrm{ECe}=8.96 \mathrm{dS} \mathrm{m}^{-1}\right)$ as presented in Tables 3-5. The data in Tables 5 and 6 report that $\mathrm{K}$ levels had significant $(p \leq 0.01)$ positive effects on all yield traits, but had no significant effects on harvest index in both two seasons. The highest $\mathrm{K}$ level $\left(\mathrm{K}_{3}=180 \mathrm{~K}_{2} \mathrm{O} \mathrm{ha}^{-1}\right)$ outperformed the other two $\mathrm{K}$ levels $\left(\mathrm{K}_{1}=60 \mathrm{~K}_{2} \mathrm{O} \mathrm{ha}^{-1}\right.$ and $\left.\mathrm{K}_{2}=120 \mathrm{~K}_{2} \mathrm{O} \mathrm{ha}^{-1}\right)$ and increased $\mathrm{Fv} / \mathrm{Fm}$ by $4.94 \%$ and $2.41 \%$ in the first season, and by $3.66 \%$ and $2.41 \%$ in the second season compared to $\mathrm{K}_{1}$ and $\mathrm{K}_{2}$, respectively. $\mathrm{K}_{3}$ also increased PI by $68.13 \%$ and $28.07 \%$ in the first season, and by $72.41 \%$ and $25.00 \%$ in the second season compared to $\mathrm{K}_{1}$ and $\mathrm{K}_{2}$, respectively. Furthermore, $\mathrm{K}_{3}$ increased SPAD values by $17.49 \%$ and $7.95 \%$ in the first season, and by $15.43 \%$ and $9.29 \%$ in the second season compared to $K_{1}$ and $K_{2}$, respectively. The highest $K$ level $\left(\mathrm{K}_{3}=180 \mathrm{~kg} \mathrm{~K}_{2} \mathrm{O} \mathrm{ha}{ }^{-1}\right)$ exceeded the other two levels of $\mathrm{K}\left(\mathrm{K}_{1}=60 \mathrm{~kg} \mathrm{~K}_{2} \mathrm{O}\right.$ ha ${ }^{-1}$ and 
$\mathrm{K}_{2}=120 \mathrm{~kg} \mathrm{~K}_{2} \mathrm{O} \mathrm{ha}^{-1}$ ) for the above growth traits. $\mathrm{K}_{3}$ increased root length by $18.12 \%$ and $9.76 \%, 17.25 \%$ and $10.26 \%$; root diameter by $18.80 \%$ and $13.01 \%, 16.00 \%$ and $12.40 \%$; root fresh weight by $19.61 \%$ and $14.35 \%, 18.75 \%$ and $13.99 \%$; top fresh weight by $29.49 \%$ and $16.09 \%, 25.88 \%$ and $17.58 \%$; and LAI by $37.32 \%$ and $27.73 \%, 35.95 \%$ and $23.86 \%$ compared to $\mathrm{K}_{1}$ and $\mathrm{K}_{2}$, in the first and second seasons, respectively. $\mathrm{K}_{3}$ increased gross sugar (\%) by $11.79 \%$ and $9.64 \%$, pure sugar $(\%)$ by $17.75 \%$ and $15.97 \%$, purity $(\%)$ by $5.08 \%$ and $5.47 \%$, and alkalinity index by $30.75 \%$ and $40.52 \%$ in the first and second seasons, respectively, compared to the $\mathrm{K}_{1}$ level. Conversely, the $\mathrm{K}_{1}$ level collected the highest $\mathrm{Na}^{+}$content (1.89 and 2.20), $\mathrm{K}^{+}$content (4.51 and 4.49), $\alpha$-amino $\mathrm{N}$ content (1.80 and 1.96), impurity index (1.85 and 1.96), and loss sugar (2.60\% and $2.77 \%)$ in the first and second seasons, respectively. Additionally, $K_{3}$ significantly exceeded the other two $K$ levels $\left(K_{1}\right.$ and $\left.K_{2}\right)$ for all yield traits, except for R-KUE ( $\mathrm{kg}$ root per $\mathrm{kg} \mathrm{K}$ ), where $\mathrm{K}_{1}$ was the best level for this trait. $\mathrm{K}_{3}$ increased root, top, biological, gross sugar, and pure sugar yields, and R-SUE by $20.80 \%$ and $17.84 \%, 23.08 \%$ and $26.27 \%, 21.22 \%$ and $19.85 \%, 34.60 \%$ and $30.24 \%, 41.76 \%$ and $37.53 \%$, and $16.67 \%$ and $12.50 \%$ in the first and second seasons, respectively, compared to the $\mathrm{K}_{1}$ level. On the other hand, the $\mathrm{K}_{1}$ level gave the maximum value of R-KUE (1.23 and $1.43 \mathrm{~kg}$ root per $\mathrm{kg} \mathrm{K}$, respectively) in both seasons.

\subsection{Effect of Sulfur Fertilization on Sugar Beet Physiological, Growth and Yield Traits}

The data in Tables $3-5$ show significant $(p \leq 0.01)$ differences among the levels of sulfur (S) for sugar beet plant Fv/Fm in the first season, and PI, SPAD, growth traits (root length and diameter, root and top fresh weight plant $^{-1}$, and LAI), and juice quality (gross sugar content, impurity index, loss sugar content, purity percentage, $\mathrm{Na}^{+}, \mathrm{K}^{+}, \alpha$-amino $\mathrm{N}$, and pure sugar content) in both seasons, but this was not true for alkalinity index in the 2019/2020 season. The data in Tables 5 and 6 show that the applied level of $S_{3}$ resulted in a significant increase in the yields of sugar beet. The responses of these traits to $S$ were gradually increased by increasing the applied $S$ level. The highest $S$ level $\left(\mathrm{S}_{3}=525 \mathrm{~kg} \mathrm{CaSO}_{4} \mathrm{ha}^{-1}\right)$ was associated with increases in Fv/Fm of $2.44 \%$ in the first season, in PI of $20.07 \%$ and $18.20 \%$, in SPAD of $11.39 \%$ and $9.91 \%$, in root length of $11.23 \%$ and $9.15 \%$, in root diameter of $19.83 \%$ and $15.32 \%$, in root fresh weight plant ${ }^{-1}$ of $19.42 \%$ and $17.19 \%$, in top fresh weight plant ${ }^{-1}$ of $29.49 \%$ and $28.92 \%$, and in LAI of $37.80 \%$ and $38.81 \%$ in the first and second seasons, respectively, compared with the lowest level $\left(\mathrm{S}_{1}=175 \mathrm{~kg} \mathrm{CaSO}_{4} \mathrm{ha}^{-1}\right)$. The $\mathrm{S}_{2}$ level $\left(350 \mathrm{~kg} \mathrm{CaSO}_{4} \mathrm{ha}^{-1}\right)$ increased gross sugar content by $2.49 \%$ and $2.99 \%$, and pure sugar content by $2.23 \%$ and $1.18 \%$ in the first and second seasons, respectively, compared to the $\mathrm{S}_{1}$ level $\left(175 \mathrm{~kg} \mathrm{CaSO}_{4}\right.$ $\mathrm{ha}^{-1}$ ). The $\mathrm{S}_{3}$ level (525 $\mathrm{kg} \mathrm{CaSO}_{4} \mathrm{ha}^{-1}$ ) increased purity (\%) by $0.79 \%$ and $2.06 \%$ in both the $2018 / 19$ and 2019/20 seasons, respectively, and alkalinity index by $46.90 \%$ in the first season compared to the $\mathrm{S}_{1}$ level. On the other hand, the $\mathrm{S}_{1}$ level collected the highest $\mathrm{Na}^{+}$content (1.81 and 2.04), $\mathrm{K}^{+}$content (4.21 and 4.22), $\alpha$-amino $\mathrm{N}$ content (1.57 and 1.62), impurity index (1.53 and 1.66), and loss sugar (2.33\% and $2.59 \%)$ in the first and second seasons, respectively. It significantly $(p \leq 0.01)$ increased root, top, biological, gross, and pure sugar yields by $8.34 \%$ and $9.60 \%, 18.58 \%$ and $14.92 \%, 10.88 \%$ and $10.87 \%, 11.77 \%$ and $13.82 \%, 11.61 \%$ and $12.00 \%$, and $6.49 \%$ and $9.52 \%$ in the first and second growing seasons, respectively, compared to $S_{1}$. On the other hand, $S_{3}$ significantly $(p \leq 0.01$ ) decreased R-SUE by $64.29 \%$ and $62.50 \%$ in the first and second seasons, respectively, and harvest index by $2.60 \%$ in the first season compared to $S_{1}$.

\subsection{Effect of the Different Two-Way Interactions of the Three Factors Studied}

For the effect of the different two-way interactions of the three factors studied (Table S1), Fv / Fm (in the first season), PI, and SPAD (in both seasons) were significantly $(p \leq 0.05$ and 0.01$)$ affected by the interaction of $\mathrm{D} \times \mathrm{K}$ levels. The interaction of $\mathrm{D}_{1} \times \mathrm{K}_{3}$ resulted in the greatest values of Fv / Fm (0.86), PI (10.19 and 10.34), and SPAD (61.48 and 62.86), respectively, compared to the other interactions. Additionally, the interaction of $\mathrm{D} \times \mathrm{S}$ levels significantly increased PI $(p \leq 0.01)$ and SPAD $(p \leq 0.05)$. The $\mathrm{D}_{1} \times \mathrm{S}_{3}$ interaction resulted in the greatest values of PI (8.84 and 9.06) and SPAD (58.47 and 59.41) in the 
first and second seasons, respectively, compared to the other interactions. The interaction of $\mathrm{K} \times \mathrm{S}$ levels significantly $(p \leq 0.01)$ affected sugar beet plant responses for SPAD in the $2019 / 2020$ season. The $K_{2} \times S_{3}$ produced the highest SPAD value (61.46), but the $\mathrm{K}_{1} \times \mathrm{S}_{1}$ produced the lowest SPAD value (47.93) compared to the other interactions. The data presented in Tables S1-S4 show that root dimensions, root and top fresh weight plant $^{-1}$, and LAI were significantly $(p \leq 0.01$ and 0.05$)$ affected by the interactions of $\mathrm{D} \times \mathrm{K}$ levels in both seasons. The interaction of $\mathrm{D}_{1} \times \mathrm{K}_{3}$ resulted in maximum values for root length $(35.48$ and $36.30 \mathrm{~cm})$, root diameter $(15.34$ and $16.05 \mathrm{~cm})$, root fresh weight (1690.3 and $\left.1657.8 \mathrm{~g} \mathrm{plant}^{-1}\right)$, top fresh weight (1.18 and $\left.1.25 \mathrm{~kg} \mathrm{plant}^{-1}\right)$, and LAI (6.76 and 6.68) in the first and second seasons, respectively. The interaction of $\mathrm{D} \times \mathrm{S}$ levels significantly $(p \leq 0.01)$ affected sugar beet growth traits with an increase in their responses as the applied $S$ level increased in both seasons. The interaction of $D_{1} \times S_{3}$ resulted in the maximum values for root length $(34.09$ and $34.79 \mathrm{~cm})$, root diameter $(15.18$ and $15.54 \mathrm{~cm}$ ), root fresh weight (1644.90 and $\left.1608.90 \mathrm{~g} \mathrm{plant}^{-1}\right)$, top fresh weight (1.17 and $1.23 \mathrm{~kg} \mathrm{plant}^{-1}$ ), and LAI (6.35 and 6.42) in the first and second seasons, respectively. The interaction of $\mathrm{K} \times \mathrm{S}$ levels significantly $(p \leq 0.01)$ affected plant growth trait in both seasons. The interaction of $\mathrm{K}_{3} \times \mathrm{S}_{3}$ resulted in maximum values for root length (35.78 and $36.44 \mathrm{~cm})$, root diameter $(16.18$ and $16.05 \mathrm{~cm})$, root fresh weight $(1701.0$ and $1694.2 \mathrm{~g}$ plant ${ }^{-1}$ ), top fresh weight (1.23 and $1.28 \mathrm{~kg} \mathrm{plant}^{-1}$ ), and LAI (7.21 and 6.92) in the first and second seasons, respectively. The data presented in Table S3 and Figures S1-S7 show that impurity index, pure sugar (\%), and purity (\%) in the 2019/2020 season, and gross sugar $(\%)$ in both the 2018/2019 and 2019/2020 seasons were significantly ( $p \leq 0.01$ and 0.05 ) affected by the interaction of $\mathrm{D} \times \mathrm{K}$ levels. The interaction of $\mathrm{D}_{1} \times \mathrm{K}_{3}$ produced the highest values of gross sugar content ( $22.6 \%$ and $22.4 \%$ in the first and second seasons, respectively) and purity (91.26\% in the second season), but the interaction of $\mathrm{D}_{2} \times \mathrm{K}_{1}$ resulted in the highest values of impurity index (2.18 in the second season). Moreover, the interactions of $\mathrm{D} \times \mathrm{S}$ levels significantly $(p \leq 0.01)$ affected alkalinity index response in the first season, while gross and pure sugar contents responded significantly in the second season. The interaction of $D_{1} \times S_{3}$ resulted in the highest gross sugar content (21.3\%) and alkalinity index (5.70), but the $D_{1} \times S_{2}$ interaction resulted in the highest pure sugar content $(19.1 \%)$ compared to the other interactions. The interaction of $\mathrm{K} \times \mathrm{S}$ levels significantly $(p \leq 0.01)$ affected sugar beet trait responses concerning gross and pure sugar contents and the impurity index in the 2019/2020 season, and significantly $(p \leq 0.05)$ affected $\mathrm{Na}^{+}$content in the $2018 / 2019$ season. The interaction of $K_{3} \times S_{3}$ produced the highest gross sugar content $(22.2 \%)$ and pure sugar content $(19.9 \%)$, the interaction of $\mathrm{K}_{1} \times \mathrm{S}_{1}$ collected the highest $\mathrm{Na}^{+}$content (2.07 meq per $100 \mathrm{~g}$ ), while the interaction of $\mathrm{K}_{1} \times \mathrm{S}_{3}$ resulted in the highest impurity index (2.19) compared with the other two-way interactions. Gross and pure sugar yields and R-KUE were significantly $(p \leq 0.01)$ increased in both the 2018/2019 and 2019/2020 seasons, as well as root yield in the second season by the interaction of $\mathrm{D} \times \mathrm{K}$ levels. Furthermore, the biological yield in both seasons, root and top yields in the first season, harvest index, and R-SUE in the second season were significantly $(p \leq 0.05)$ influenced by D $\times$ K levels (Tables S9 and S10 and Figures S8-S19). The interaction of $\mathrm{D}_{1} \times \mathrm{K}_{3}$ gave the highest root yield (92.72 and $91.89 \mathrm{Mg} \mathrm{ha}^{-1}$ ), top yield (33.77 and $34.94 \mathrm{Mg} \mathrm{ha}^{-1}$ ), biological yield (126.47 and $126.83 \mathrm{Mg} \mathrm{ha}^{-1}$ ), gross sugar yield (20.97 and $\left.20.63 \mathrm{Mg} \mathrm{ha}^{-1}\right)$, pure sugar yield (19.23 and $\left.18.83 \mathrm{Mg} \mathrm{ha}^{-1}\right)$, and R-SUE (0.30 kg root per $\mathrm{kg} \mathrm{S}$ ), while the lowest R-KUE (1.35 and $1.61 \mathrm{~kg}$ root per $\mathrm{kg} \mathrm{K}$ ) was produced by the interaction of $D_{1} \times K_{1}$ and the lowest harvest index (0.78) was produced by the interaction of $\mathrm{D}_{2} \times \mathrm{K}_{1}$ compared to the other interactions. Root, biological, gross, and pure sugar yields, R-KUE, and R-SUE in both seasons, as well as the harvest index in the second season were significantly $(p \leq 0.01)$ influenced by the interaction of $\mathrm{D} \times \mathrm{S}$ levels. Additionally, top yield was significantly $(p \leq 0.05)$ influenced in the first season by the same interaction (Tables S3 and S4; Figures S8-S19). The interaction of $\mathrm{D}_{1} \times \mathrm{S}_{3}$ significantly collected the highest root yield (88.61 and $88.16 \mathrm{Mg} \mathrm{ha}^{-1}$ ), top yield (33.13 and $34.22 \mathrm{Mg}$ $\mathrm{ha}^{-1}$ ), biological yield (121.74 and $122.38 \mathrm{Mg} \mathrm{ha}^{-1}$ ), gross sugar yield (18.92 and $18.97 \mathrm{Mg}$ 
$\mathrm{ha}^{-1}$ ), pure sugar yield (16.99 and $\left.16.85 \mathrm{Mg} \mathrm{ha}^{-1}\right)$, and R-KUE (0.90 and $1.54 \mathrm{~kg}$ root per $\mathrm{kg} \mathrm{K})$. However, the lowest R-SUE ( 0.45 and $0.44 \mathrm{~kg}$ root per $\mathrm{kg} \mathrm{S}$ ) were produced with the interaction of $D_{1} \times S_{1}$ and the lightest harvest index (0.77) was produced with the interaction of $\mathrm{D}_{2} \times \mathrm{S}_{1}$ in the first and second seasons, respectively, compared to the other two-way interactions. Root, biological, gross, and pure sugar yields, R-KUE, and R-SUE in the 2018/2019 and 2019/2020 seasons, as well as top yield and harvest index in the second season and top yield in the first season, were significantly $(p \leq 0.01$ and 0.05$)$ influenced by the interaction of $\mathrm{K} \times \mathrm{S}$ levels (Tables S3 and S4; Figures S8-S19). The interaction of $\mathrm{K}_{3} \times \mathrm{S}_{3}$ resulted in the significant highest root yield ( 91.73 and $89.11 \mathrm{Mg} \mathrm{ha}^{-1}$ ), top yield (30.46 and $32.44 \mathrm{Mg} \mathrm{ha}^{-1}$ ), biological yield (122.17 and $121.55 \mathrm{Mg} \mathrm{ha}^{-1}$ ), gross sugar yield (20.25 and $\left.19.97 \mathrm{Mg} \mathrm{ha}^{-1}\right)$, and pure sugar yield (18.37 and $\left.17.94 \mathrm{Mg} \mathrm{ha}^{-1}\right)$. However, the lowest R-KUE (1.26 and $1.56 \mathrm{~kg}$ root per $\mathrm{kg} \mathrm{K}$ ) was obtained with the interaction of $\mathrm{D}_{1} \times \mathrm{S}_{3}$, and the maximum R-SUE ( 0.45 and $0.43 \mathrm{~kg}$ root per $\mathrm{kg} \mathrm{S}$ ) was produced with the interaction of $D_{3} \times S_{1}$ in the first and second growing seasons, respectively, compared to the other two-way interactions.

\subsection{Effect of the Different Three-Way Interactions of the Three Factors Studied}

Concerning the effect of the applied three-way interactions, Tables 3 and 4 display significant $(p \leq 0.01)$ differences by the interaction of $\mathrm{D}_{1} \times \mathrm{K}_{3} \times \mathrm{S}_{3}$ for the growth traits mentioned above in both seasons. The data listed in Tables 4 and 5 show that significant $(p \leq 0.01)$ differences were reported for gross and pure sugar contents in the 2019/2020 season. The interaction of $\mathrm{D}_{1} \times \mathrm{K}_{3} \times \mathrm{S}_{3}$ gave the maximum values of all juice quality traits compared with the other three-way interactions. The results in Tables 5 and 6 show significant $(p \leq 0.01)$ variations for root, top, biological, gross, and pure sugar yields, R-KUE, and R-SUE in both seasons, and harvest index in the second season. The results in Figures S20 and S21 show that the interaction of $D_{1} \times K_{3} \times S_{3}$ gave the highest root yield (104.91 and $104.39 \mathrm{Mg} \mathrm{ha}^{-1}$ ) and pure sugar yield (21.85 and $22.16 \mathrm{Mg} \mathrm{ha}^{-1}$ ) in the first and second seasons, respectively, compared to the other three-way interactions.

\subsection{The Direct and Indirect, Stepwise Regression, and Correlation Analyses}

The data in Table 7 show that, under soil salinity $\left(\mathrm{ECe}=8.96 \mathrm{dS} \mathrm{m}^{-1}\right)$ conditions, the direct (bolded and underlined values) and indirect effects of seven specified pure sugar yield (dependent variable) are related traits to pure sugar yield. The data of path coefficient analysis show that the root yield, pure sugar content, SPAD values, and LAI for sugar beet had positive direct effects with 0.62 and $0.65,0.38$ and $0.38,0.01$ and 0.0041 , and 0.05 and 0.05 path coefficients in the first and second seasons, respectively, on pure sugar yield. Root length, root diameter, and root fresh weight traits had the highest indirect positive effects on pure sugar yield through root yield by 0.60 and $0.54,0.60$ and 0.54 , and 0.61 and 0.57 in the 2018/2019 and 2019/2020 seasons, respectively. Furthermore, pure sugar content and SPAD values had the greatest indirect positive effects on pure sugar yield through root yield by 0.58 and 0.49 , and 0.45 and 0.48 in the first and second seasons, respectively. In Table 8 , correlation and regression data analysis between pure sugar yield and each of pure sugar content, root dimensions, root fresh weight, root yield, LAI, and SPAD values were calculated to concentrate on the relationship of the efficacious sugar beet traits interest. Highly significant $(p \leq 0.01)$ positive correlations were found between pure sugar yield and root yield $\left(\mathrm{r}=0.966^{* *}\right.$ and $\left.0.958^{* *}\right)$ and between the dependent variable and each of pure sugar content $\left(\mathrm{r}=0.909^{* *}\right.$ and $\left.0.866^{* *}\right)$, root length $\left(\mathrm{r}=0.907^{* *}\right.$ and $\left.0.944^{* *}\right)$, and SPAD values $\left(r=0.820^{* *}\right.$ and $\left.0.983^{* *}\right)$. Furthermore, highly significant positive correlations $\left(\mathrm{r}=0.921^{* *}\right.$ and $0.937^{* *}, \mathrm{r}=0.869^{* *}$ and $920^{* *}$ and $\mathrm{r}=0.876^{* *}$ and $\left.0.925^{* *}\right)$ were observed between root yield and each of root length, root diameter, and root fresh weight in the first and second seasons, respectively. The stepwise regression in Table 9 shows the significant $(p \leq 0.01)$ contribution of three traits (i.e., root yield, pure sugar content, and LAI) to the variations in pure sugar yield. 


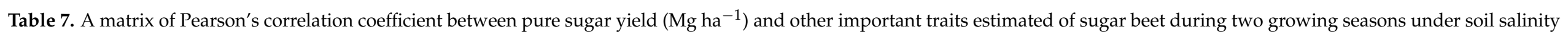
$\left(\mathrm{ECe}=8.96 \mathrm{dS} \mathrm{m}^{-1}\right)$ conditions.

\begin{tabular}{|c|c|c|c|c|c|c|c|c|c|c|c|c|c|c|c|c|}
\hline \multirow[t]{2}{*}{ Character } & \multicolumn{2}{|c|}{$\begin{array}{l}\text { Pure Sugar } \\
\text { Yield } \\
\left(\mathrm{Mg} \mathrm{ha}^{-1}\right)\end{array}$} & \multicolumn{2}{|c|}{$\begin{array}{c}\text { Pure Sugar } \\
\text { Content }\end{array}$} & \multicolumn{2}{|c|}{$\begin{array}{l}\text { Root Length } \\
\text { (cm) }\end{array}$} & \multicolumn{2}{|c|}{$\begin{array}{l}\text { Root Diameter } \\
\quad \text { (cm) }\end{array}$} & \multicolumn{2}{|c|}{$\begin{array}{l}\text { Root Fresh } \\
\text { Weight } \\
\left.\text { (kg plant }^{-1}\right)\end{array}$} & \multicolumn{2}{|c|}{$\begin{array}{l}\text { Root Yield } \\
\left(\mathrm{Mg} \mathrm{ha}^{-1}\right)\end{array}$} & \multicolumn{2}{|c|}{ LAI } & \multicolumn{2}{|c|}{ SPAD } \\
\hline & 2018/19 & $2019 / 20$ & $2018 / 19$ & $2019 / 20$ & $2018 / 19$ & $2019 / 20$ & $2018 / 19$ & $2019 / 20$ & $2018 / 19$ & $2019 / 20$ & 2018/19 & $2019 / 20$ & 2018/19 & $2019 / 20$ & 2018/19 & $2019 / 20$ \\
\hline $\begin{array}{l}\text { Pure sugar yield } \\
\left(\mathrm{Mg} \mathrm{ha}^{-1}\right)\end{array}$ & 1 & 1 & $0.909 * *$ & $0.866^{* *}$ & $0.907^{* *}$ & $0.944^{* *}$ & $0.837^{* *}$ & $0.920 * *$ & $0.861^{* *}$ & $0.921 * *$ & $0.966^{* *}$ & $0.958^{* *}$ & $0.783^{* *}$ & $0.790 * *$ & $0.820^{* *}$ & $0.983^{* *}$ \\
\hline Pure sugar content & & & 1 & 1 & $0.740 * *$ & $0.756^{* *}$ & $0.639 * *$ & $0.711^{* *}$ & $0.691^{* *}$ & $0.684^{* *}$ & $0.778^{* *}$ & $0.694^{* *}$ & $0.669^{* *}$ & $0.620 * *$ & 0.728 ** & $0.706^{* *}$ \\
\hline Root length (m) & & & & & 1 & 1 & 0.881 ** & $0.921 * *$ & $0.886^{* *}$ & $0.947^{* *}$ & $0.921 * *$ & $0.937^{* *}$ & $0.827^{* *}$ & $0.827 * *$ & $0.878^{* *}$ & $0.893^{* *}$ \\
\hline Root diameter $(\mathrm{cm})$ & & & & & & & 1 & 1 & $0.834^{* *}$ & $0.960 * *$ & $0.869 * *$ & $0.920 * *$ & $0.849 * *$ & $0.908^{* *}$ & 0.773 ** & $0.910^{* *}$ \\
\hline Root FW (kg plant ${ }^{-1}$ ) & & & & & & & & & 1 & 1 & $0.876^{* *}$ & $0.925^{* *}$ & $0.783^{* *}$ & $0.894^{* *}$ & $0.765^{* *}$ & $0.885^{* *}$ \\
\hline Root yield $\left(\mathrm{Mg} \mathrm{ha}^{-1}\right)$ & & & & & & & & & & & 1 & 1 & $0.749 * *$ & $0.765^{* *}$ & $0.789 * *$ & $0.885^{* *}$ \\
\hline LAI & & & & & & & & & & & & & 1 & 1 & $0.783^{* *}$ & $0.849^{* *}$ \\
\hline SPAD & & & & & & & & & & & & & & & 1 & 1 \\
\hline
\end{tabular}

${ }^{* *} p \leq 0.01$, and ns; not significant. FW $=$ fresh weight. 


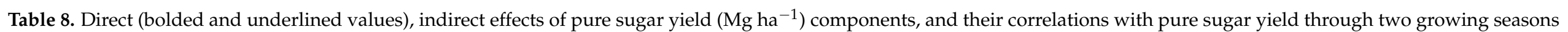
under soil salinity $\left(\mathrm{ECe}=8.96 \mathrm{dSm}^{-1}\right)$ conditions.

\begin{tabular}{|c|c|c|c|c|c|c|c|c|c|c|c|c|c|c|}
\hline \multirow[t]{2}{*}{ Character } & \multicolumn{2}{|c|}{$\begin{array}{l}\text { Root Yield } \\
\left(\mathrm{Mg} \mathrm{ha}^{-1}\right)\end{array}$} & \multicolumn{2}{|c|}{$\begin{array}{c}\text { Pure Sugar } \\
\text { Content }\end{array}$} & \multicolumn{2}{|c|}{ Root Length (cm) } & \multicolumn{2}{|c|}{$\begin{array}{l}\text { Root Diameter } \\
\text { (cm) }\end{array}$} & \multicolumn{2}{|c|}{$\begin{array}{c}\text { Root FW } \\
\left(\text { kg plant }^{-1}\right)\end{array}$} & \multicolumn{2}{|c|}{ SPAD Values } & \multicolumn{2}{|c|}{ LAI } \\
\hline & 2018/19 & $2019 / 20$ & 2018/19 & $2019 / 20$ & 2018/19 & $2019 / 20$ & 2018/19 & $2019 / 20$ & 2018/19 & $2019 / 20$ & 2018/19 & $2019 / 20$ & $2018 / 19$ & $2019 / 20$ \\
\hline Root yield $\left(\mathrm{Mg} \mathrm{ha}^{-1}\right)$ & 0.50 & 0.46 & 0.58 & 0.49 & 0.60 & 0.54 & 0.60 & 0.54 & 0.61 & 0.57 & 0.45 & 0.48 & $\underline{0.65}$ & $\underline{0.62}$ \\
\hline Pure sugar content & 0.23 & 0.26 & 0.27 & 0.28 & 0.26 & 0.27 & 0.27 & 0.24 & 0.28 & 0.28 & $\underline{0.38}$ & $\underline{0.38}$ & 0.26 & 0.30 \\
\hline Root length $(\mathrm{cm})$ & 0.0035 & -0.02 & 0.0038 & -0.02 & 0.0040 & -0.02 & 0.0039 & -0.02 & $\underline{0.0042}$ & -0.02 & 0.0032 & -0.01 & 0.004 & -0.02 \\
\hline Root diameter $(\mathrm{cm})$ & 0.0015 & -0.0003 & 0.0015 & -0.0003 & 0.0016 & -0.0003 & $\underline{0.0017}$ & -0.0004 & 0.0015 & -0.0004 & 0.0012 & -0.0003 & 0.0015 & -0.0004 \\
\hline SPAD values & 0.0035 & 0.01 & $\underline{0.0041}$ & $\underline{0.01}$ & 0.0036 & 0.01 & 0.0037 & 0.01 & 0.0036 & 0.01 & 0.0029 & 0.01 & 0.0036 & 0.01 \\
\hline LAI & $\underline{0.05}$ & $\underline{0.05}$ & 0.05 & 0.04 & 0.05 & 0.04 & 0.05 & 0.05 & 0.05 & 0.04 & 0.03 & 0.04 & 0.04 & 0.04 \\
\hline Total $r$ & 0.79 ** & $0.78^{* *}$ & $0.98^{* *}$ & $0.82^{* *}$ & $0.91^{* *}$ & $0.86^{* *}$ & $0.92 * *$ & $0.84^{* *}$ & 0.94 & 0.91 ** & $0.87 * *$ & $0.91^{* *}$ & $0.96^{* *}$ & $0.97^{* *}$ \\
\hline
\end{tabular}


Table 9. Correlation coefficient (r), coefficient of determination $\left(R^{2}\right)$, and standard error of the estimates (SEE) for predicting pure sugar yield $\left(\mathrm{Mg} \mathrm{ha}^{-1}\right)$ in two growing seasons under soil salinity $\left(\mathrm{ECe}=8.96 \mathrm{~d} \mathrm{Sm}^{-1}\right)$ conditions.

\begin{tabular}{|c|c|c|c|c|}
\hline Season & $\mathbf{R}^{2}$ & SEE & Seg. & Fitted Equation \\
\hline 2018/20190.999 & 0.998 & 0.062 & ** & Pure sugar yield $=-5.859+0.182$ root yield +0.309 pure sugar $\%+0.06 \mathrm{LAI}$ \\
\hline 2019/20200.999 & 0.997 & 0.068 & ** & Pure sugar yield $=-5.937+0.183$ root yield +0.310 pure sugar $\%+0.06 \mathrm{LAI}$ \\
\hline
\end{tabular}

\section{Discussion}

In the present study, in addition to soil salinity (ECe $=8.96 \mathrm{dS} \mathrm{m}^{-1}$; Table 1), its potassium $\left(\mathrm{K}^{+}\right)$content is $39.124 \mathrm{mg} \mathrm{kg}^{-1}$ soil, making it $\mathrm{K}^{+}$-poor soil. Additionally, saline soils often suffer from a deficiency of nutrients, including $S$ [27], so the soil examined in this study is deficient in S. Therefore, it was necessary to supply the tested saline soil with sufficient amounts of $\mathrm{K}^{+}$(to antagonize the harmful $\mathrm{Na}^{+}$ion) and $\mathrm{S}$ in favor of sugar beet plants to be able to take their nutritional requirements to be robust and thus be able to resist/tolerate the soil salinity conditions. In addition to the nutritional factor, the key climatic factor affecting sugar beet productivity is temperature. For sugar beet plants to obtain sufficient thermal units throughout their growing season, it has been estimated the seasonal growing period of sugar beet plants to be approximately 200 days [45]. Thus, the sowing date of sugar beet has a great influence on the plant development and productivity through the adequate accumulation of thermal units, especially from the emergence stage until sugar beet plants reach the harvest stage [45]. Therefore, the date of sowing beets should have been early on the 1st of September to meet the required growth period for the plants to be supplied with the required thermal units, which would be reflected in the best growth and high yield with high quality under the tested salty soil conditions.

For the above reasons, the saline soil examined in this study was provided with three levels of $\mathrm{K}\left(\mathrm{K}_{1}=60, \mathrm{~K}_{2}=120\right.$, and $\left.\mathrm{K}_{3}=180 \mathrm{~kg} \mathrm{~K}_{2} \mathrm{O} \mathrm{ha}^{-1}\right)$ and/or $\mathrm{S}\left(\mathrm{S}_{1}=175\right.$, $\mathrm{S}_{2}=350$, and $\mathrm{S}_{3}=525 \mathrm{~kg} \mathrm{CaSO}_{4} \mathrm{ha}^{-1}$ ), along with early sugar beet sowing on the 1st of September to provide all somewhat better environmental conditions for seed germination and seedling/plant growth until harvest to obtain the preferable yield with high quality for sugar beet plants grown under the adverse conditions of soil salinity.

The increased values recorded for Fv / Fm and PI (photosynthetic efficiency), along with SPAD (chlorophyll concentration) (Table 3), may be due to better climatic conditions with early sowing on the 1st of September which allowed plants to accumulate the maximum thermal units (GDD $=3274.25$ and 3392.75) compared to the late sowing date on the 1st of October (GDD = 3047.45 and 3175.55) in the 2018/2019 and 2019/2020 seasons, respectively (Table 2). This finding allows for optimal early emergence, plant development, and leaf surface area, especially in the early stage of sugar beet plants. These increases in chlorophyll concentration and photosynthetic efficiency with $\mathrm{K}$ and $\mathrm{S}$ applications (especially the highest levels) $[3,27]$ along with the early sowing date enabled plants to photosynthesize for more assimilates to obtain the highest root yield with high quality (Tables 3-6) under salt stress conditions. The increase in root dimensions, root and top fresh weight, and LAI may be due to the exposure of sugar beet plants to some favorable environmental conditions prevalent during the late growth stage and may be due to the increase in chlorophyll concentration and photosynthetic efficiency (Fv /Fm, PI, and SPAD), which resulted from the early sowing date employing accumulate higher thermal units for efficient photosynthesis increase leaf surface area to improve plant growth and increase the root weight of sugar beet [46].

The early sowing date (the 1st of September) also resulted in a marked increase in juice quality traits compared to the late sowing (the 1st of October) (Tables 4-6). These improvements in juice quality characteristics can be attributed to the appropriate climatic conditions, particularly the light and temperature required for the plant to perform well concerning the effective photosynthesis process [47]. This investigation reported a positive 
response of the photosynthesis process to the effective temperature with sufficient light, water (with increasing the osmolyte $\mathrm{K}^{+}$by $\mathrm{K}$ application), and nutrients (acidification of growing medium with increasing $\mathrm{S}$ by $\mathrm{S}$ application) in favor of adequate chlorophyll concentration and photosynthetic efficiency (Table 3) for efficient production with high quality of sugar beet plants. The early sowing date resulted in a marked increase in gross and pure sugar contents, purity percentage, and alkalinity index, while it resulted in a marked decrease in impurities (e.g., $\mathrm{Na}^{+}, \mathrm{K}^{+}$, and $\alpha$-amino $\mathrm{N}$ ), impurity index, and loss sugar percentage (Tables 4 and 5). Some reports indicated that the early sowing of sugar beet markedly increases the sugar content, purity percentage, and pure sugar content $[11,13]$. Moreover, it was reported a positive correlation between climatic factors and sugar beet giving yield quality traits [48]. These findings can be attributed to that early sowing enables sugar beet plants to collect maximum energy for storing sugars in tubers. Besides, early sowing is likely to result in more possibilities for more favorable plant growth. These results are confirmed by those in [13,46,49]. Furthermore, the root, top, biological, gross sugar, and pure sugar yields, as well as R-KUE and R-SUE were markedly increased with the early sowing. These results can be attributed to better climatic conditions conferring higher chlorophyll concentration and photosynthetic efficiency ( $\mathrm{Fv} / \mathrm{Fm}, \mathrm{PI}$, and SPAD) that encouraged increased leaf surface area, root dimensions, root and top fresh weights, and gross and pure sugar contents, which reflected in the increased root, top, and biological yields, thus increasing the pure sugar yield. These results are confirmed by those in $[13,46,49]$.

Merwad et al. [26] reported that increased salt tolerance in sugar beet plants has been correlated with increased $\mathrm{K}^{+}$availability in plant tissues. Salt damage in plants can be prevented by increasing the $\mathrm{K}^{+}$content due to its beneficial roles, directly as a protective osmoprotectant and indirectly by being used in antioxidation [50]. The beneficial effects of fertilization with $\mathrm{K}^{+}$, especially the highest level $\left(180 \mathrm{~kg} \mathrm{~K}_{2} \mathrm{O}\right.$ ha $\left.{ }^{-1}\right)$, of sugar beet plants growing under salt stress are related to its key roles in photosynthesis, protein synthesis, photosynthates translocation, control of ionic balance, and water availability [21]. Some investigations have confirmed, under salt stress, the importance of applying $\mathrm{K}^{+}$alone or in combinations in improving enzymatic activities, causing increased nutrient mobilization in the plant and translocation of photo-assimilates to active growing organs in the plant system to improve plant growth and high-quality production, all due to the improved chlorophyll concentration and photosynthetic efficiency (Fv /Fm, PI, and SPAD) [26,51,52]. Sufficient $\mathrm{K}^{+}$supply to saline soil $\left(\mathrm{ECe}=8.96 \mathrm{dS} \mathrm{m}^{-1}\right)$ increases chlorophyll concentration (SPAD value) and leaf photosynthetic carbon, and thus also enhances light reaction routes (PSI and PSII) [53], which are strongly reflected in the increased growth and high-quality productivity for sugar beet plants. In this study, although applying $\mathrm{K}^{+}$to the examined salty soil increased the gross and pure sugar contents, quality percentage, and alkalinity index in the tubers of sugar beet, it markedly decreased impurities such as $\mathrm{Na}^{+}, \mathrm{K}^{+}, \alpha-$ amino $\mathrm{N}$, impurity index, and loss sugar content, which may be attributed to the major role of $\mathrm{K}^{+}$in stimulating starch synthetase enzymes and the accumulation of carbohydrates that transfer from leaves to developing tubers of sugar beet, thus improving biochemical traits [54]. The major role of $\mathrm{K}^{+}$in inducing enzymatic activity and photosynthesis process is correlated to the synthesis of sucrose and the carrying of photosynthesized sucrose to phloem to raise sugar's level [55]. Mehrandish et al. [21] illustrated that applying $\mathrm{K}^{+}$ increases recoverable sugar and reduces impurity traits. Moreover, increasing top and biological yields associate with increasing Fv/Fm, PI, SPAD value, top fresh weight, and leaf area index. These results confirm the results of this study. Table 6 shows that gross (root yield multiplied by gross sugar content) and pure sugar (root yield multiplied by pure sugar content) yields increased due to using $\mathrm{K}^{+}$for salty soil can be explained by the fact that $\mathrm{K}^{+}$plays a major role in enhancing all plant morpho-physiology, root yield, and gross and pure sugar contents, thus increasing gross and pure sugar yields of sugar beet plants. Additionally, the highest value of R-KUE was obtained using the highest $\mathrm{K}^{+}$ that can be attributed to the highest $\mathrm{K}^{+}$level was associated with the highest increase in 
root yield. Furthermore, the highest R-SUE was obtained when sugar beet plants received $525 \mathrm{~kg} \mathrm{CaSO}_{4} \mathrm{ha}^{-1}$ to give the highest root yield.

The inclusion of $S$ in the plant's stress defense system increases plant tolerance to stresses, including salinity [27], and thus the application of $S$ to saline soil alone or in combinations increased chlorophyll concentration and photosynthetic efficiency ( $\mathrm{Fv} / \mathrm{Fm}$, PI, and SPAD values), which were reflected in the increased performance of sugar beet plants under salt stress (Tables 3-6). A report [27] indicates that applying $S$ to salt-stressed plants increases glutathione pool (a compound containing S), which may lead to increased photosynthesis efficiency due to the critical role of $S$ in photosynthesis functions and the improvement of the leaf chlorophyll concentration, thus increasing plant growth and yield characteristics. In this study, a gradual increase in S level from 175 to $525 \mathrm{~kg} \mathrm{CaSO}_{4} \mathrm{ha}^{-1}$ to salt-stressed sugar beet plants resulted in a gradual increase in chlorophyll concentration and photosynthetic efficiency (Fv/Fm, PI, and SPAD values), which were reflected in increased plant growth and yield characteristics with high quality. These positive results can also be attributed to that adding $S$ to the soil may be enhanced soil properties and fertility in favor of the growing plants [27], causing an increase in the photosynthetic area in sugar beet plants that it gives maximum returns. Additionally, the impurity traits $(\mathrm{Na}$, $\mathrm{K}$, and $\alpha$-amino $\mathrm{N}$ ), impurity index, and loss sugar content were decreased by applying $\mathrm{S}$, especially the highest level, thus increasing the gross and pure sugar content with high purity and quality percentages, and alkalinity index, while non-sugars decreased in saltstressed sugar beet plants (Tables 4 and 5). The increase in yield traits and their quality can be illustrated based on the increases in growth traits, which in turn were achieved through increased chlorophyll concentration and photosynthesis efficiency, all due to the beneficial effects of $\mathrm{S}$ applied alone or in combinations (Tables 3-6).

Fertilizing strategy of saline soils (ECe $=8.96 \mathrm{dS} \mathrm{m}^{-1}$ ) is very important to bring the nutrients into balance status in favor of growing plants. Applying $\mathrm{K}^{+}$up to $180 \mathrm{~kg}$ $\mathrm{K}_{2} \mathrm{O} \mathrm{ha}^{-1}$ with S application to these defective soils up to $525 \mathrm{~kg} \mathrm{CaSO}_{4} \mathrm{ha}^{-1}$ can help, as effective agronomical practice, these defective soils to become highly productive due to overcoming the effects of high salinity and encouraging increased salt tolerance in sugar beet to improve its productivity and industrial traits by increasing K- and S-use efficiency under dry environmental conditions.

Finally, various environmental foes, including the foes studied in this work, have negative impacts on plant growth. These negative impacts may exceed the natural tolerance capacity of stressed plants. In this case, the components of the stressed plant's defense system do not meet the requirements of adequate defense, and therefore the external use of auxiliary substances such as nutrients and other beneficial strategies helps the plants to increase the efficiency of their antioxidant defenses; thus, plants can perform efficiently under adverse conditions of environmental foes [3,4,8,56-59].

\section{Conclusions}

This work was conducted to shed light on the potential positive effects of potassium and sulfur applied to saline soil ( $\mathrm{ECe}=8.96 \mathrm{dS} \mathrm{m}^{-1}$ ) to stimulate salt tolerance by promoting growth, pure sugar yield, and juice quality, as well as K- and S-use efficiency of sugar beet with two sowing dates in semi-arid regions. Early sowing date (the 1st of September) increased responses of morpho-physiological trait responses (root dimensions and weight; top fresh weight; Fv /Fm; PI; SPAD; LAI; juice quality; productivity; root, top, biological, gross, and pure sugar yields; and the alkalinity index; as well as $\mathrm{K}$ - and $\mathrm{S}$-use efficiency) to soil fertilization with potassium (especially at a level of $180 \mathrm{~kg} \mathrm{~K}{ }_{2} \mathrm{O}$ $\mathrm{ha}^{-1}$ ) and sulfur (especially at a level of $525 \mathrm{~kg} \mathrm{CaSO}_{4} \mathrm{ha}^{-1}$ ) under salt stress conditions. The application of potassium and sulfur induced salt tolerance in sugar beet plants by enhancing growth indices and sugar quality traits while reducing impurity traits $(\mathrm{Na}, \mathrm{K}$, and $\alpha$-amino $\mathrm{N}$ ), loss sugar content, and impurity index. Path coefficient analysis data showed that root yield, pure sugar content, SPAD, and LAI in sugar beet had positive direct effects with 0.62 and $0.65,0.38$ and $0.38,0.01$ and 0.0041 , and 0.05 and 0.05 path coeffi- 
cients, in the first and second seasons, respectively, on pure sugar yield. Highly significant $(p \leq 0.01)$ positive correlations were found between pure sugar yield and root yield $\left(\mathrm{r}=0.966^{* *}\right.$ and $\left.0.958^{* *}\right)$, and between the dependent variable and each of pure sugar content $\left(\mathrm{r}=0.909^{* *}\right.$ and $\left.0.866^{* *}\right)$, root length $\left(\mathrm{r}=0.907^{* *}\right.$ and $\left.0.944^{* *}\right)$, and SPAD value $\left(r=0.820^{* *}\right.$ and $\left.0.983^{* *}\right)$. Stepwise regression data showed that three traits (i.e., root yield, pure sugar $(\%)$, and LAI) contributed significantly $(p \leq 0.001)$ to the variations in pure sugar yield. Soil application with potassium and sulfur with the above-mentioned doses can be assisted to correct their reductions in the saline soils to reduce salt stress effects on sugar beet plants. The results of our study will open new research prospects for fertilization strategy, one of the important factors for overcoming different abiotic stresses, especially salinity in climate change scenarios.

Supplementary Materials: The following are available online at https:/ /www.mdpi.com/article/10 .3390/agronomy11040806/s1, Table S1: Photosynthetic efficiency (Fv/Fm, PI(\%), and SPAD values), and root length $(\mathrm{cm})$ of sugar beet as affected by the interactions of sowing date (D), potassium $(\mathrm{K})$ and sulphur (S), during two growing seasons (Mean $\pm \mathrm{SE}$ ) under soil salinity (ECe $=8.96 \mathrm{dS} \mathrm{m}^{-1}$ ) conditions, Table S2: Root diameter, root fresh weight, and top fresh weight of sugar beet as affected by the interactions of sowing date (D), potassium (K) and sulphur (S), during two growing seasons (Mean $\pm \mathrm{SE}$ ) under soil salinity $\left(\mathrm{ECe}=8.96 \mathrm{dS} \mathrm{m}^{-1}\right.$ ) conditions, Table S3: Leaf area index (LAI), biological yield, harvest index, and purity content of sugar beet as affected by the interactions of sowing date (D), potassium (K) and sulphur (S), during two growing seasons (Mean $\pm \mathrm{SE}$ ) under soil salinity $\left(\mathrm{ECe}=8.96 \mathrm{dS} \mathrm{m}^{-1}\right)$ conditions, Table S4: Root yield, and top yield of sugar beet as affected by the interactions of sowing date (D), potassium $(\mathrm{K})$ and sulphur (S), during two growing seasons (Mean $\pm \mathrm{SE}$ ) under soil salinity $\left(\mathrm{ECe}=8.96 \mathrm{dS} \mathrm{m}^{-1}\right.$ ) conditions, Figure S1: $\mathrm{Na}^{+}$(meq per $100 \mathrm{~g}$ ) in 2018/2019 season and impurity index in 2019/2020 season of sugar beet as affected by the interactions of sowing dates (D) and potassium $(\mathrm{K})$, (Mean $\pm \mathrm{SE}$ ) under soil salinity $\left(\mathrm{ECe}=8.96 \mathrm{dS} \mathrm{m}^{-1}\right.$ ) conditions, Figure S2: $\mathrm{Na}^{+}$(meq per $100 \mathrm{~g}$ ) in 2018/2019 season and impurity index in 2019/2020 season of sugar beet as affected by the interactions of potassium $(\mathrm{K})$ and sulphure (S), (Mean $\pm \mathrm{SE}$ ) under soil salinity $\left(\mathrm{ECe}=8.96 \mathrm{dS} \mathrm{m}^{-1}\right)$ conditions, Figure S3: Gross sugar $(\%)$ and alkalinity index of sugar beet as affected by the interactions of sowing dates (D) and potassium (K), in 2018/2019 season (Mean \pm SE) under soil salinity (ECe $=8.96 \mathrm{dS} \mathrm{m}^{-1}$ ) conditions, Figure S4: Gross and pure sugar (\%) of sugar beet as affected by the interactions of sowing dates (D) and potassium (K), in 2019/2020 season (Mean \pm SE) under soil salinity $\left(\mathrm{ECe}=8.96 \mathrm{dS} \mathrm{m}^{-1}\right)$ conditions, Figure S5: Gross sugar $(\%)$ and alkalinity index of sugar beet as affected by the interactions of sowing dates (D) and sulphure (S),in 2018/2019 season, (Mean \pm SE) under soil salinity $\left(\mathrm{ECe}=8.96 \mathrm{dS} \mathrm{m}^{-1}\right)$ conditions, Figure S6: Gross and pure sugar $(\%)$ of sugar beet as affected by the interactions of sowing dates (D) and sulphure (S), in 2019/2020 season (Mean \pm SE) under soil salinity $\left(\mathrm{ECe}=8.96 \mathrm{dS} \mathrm{m}^{-1}\right.$ ) conditions, Figure S7: Gross and pure sugar (\%) of sugar beet as affected by the interactions of potassium (K) and sulphure (S), in 2019/2020 season (Mean \pm SE) under soil salinity $\left(E C e=8.96 \mathrm{dS} \mathrm{m}^{-1}\right)$ conditions, Figure S8: Gross and pure sugar yield $\left(\mathrm{t} \mathrm{h}^{-1}\right)$ of sugar beet as affected by the interactions of sowing dates (d) and potassium (K), in 2018/2019 season (Mean \pm SE) under soil salinity $\left(E C e=8.96 \mathrm{dS} \mathrm{m}^{-1}\right)$ conditions, Figure S9: Gross and pure sugar yield $\left(\mathrm{t} \mathrm{h}^{-1}\right)$ of sugar beet as affected by the interactions of sowing dates (d) and potassium (K), in 2019/2020 season (Mean $\pm \mathrm{SE}$ ) under soil salinity $\left(\mathrm{ECe}=8.96 \mathrm{dS} \mathrm{m}^{-1}\right)$ conditions, Figure S10: Gross and pure sugar yield $\left(\mathrm{Mg} \mathrm{h}^{-1}\right)$ of sugar beet as affected by the interactions of sowing dates (d) and sulphure (S), in 2018/2019 season (Mean $\pm \mathrm{SE}$ ) under soil salinity $\left(\mathrm{ECe}=8.96 \mathrm{dS} \mathrm{m}^{-1}\right.$ ) conditions, Figure S11: Gross and pure sugar yield $\left(\mathrm{Mg} \mathrm{h}^{-1}\right)$ of sugar beet as affected by the interactions of sowing dates (d) and sulphure (S), in 2019/2020 season (Mean \pm SE) under soil salinity $\left(E C e=8.96 \mathrm{dS} \mathrm{m}^{-1}\right.$ ) conditions, Figure S12: Gross and pure sugar yield $\left(\mathrm{Mg} \mathrm{h}^{-1}\right)$ of sugar beet as affected by the interactions of potassium $(\mathrm{K})$ and sulphure $(\mathrm{S})$, in 2018/2019 season (Mean \pm SE) under soil salinity $\left(\mathrm{ECe}=8.96 \mathrm{dS} \mathrm{m}^{-1}\right)$ conditions, Figure S13: Gross and pure sugar yield $\left(\mathrm{Mg} \mathrm{h}^{-1}\right)$ of sugar beet as affected by the interactions of potassium (K) and sulphure (S), in 2019/2020 season (Mean \pm SE) under soil salinity $\left(E C e=8.96 \mathrm{dS} \mathrm{m}^{-1}\right.$ ) conditions, Figure S14: R-KUE ( $\mathrm{kg}$ root $\mathrm{kg} \mathrm{K}^{-1}$ ) and R-SUE $\left(\mathrm{kg}\right.$ root $\mathrm{kg} \mathrm{S}^{-1}$ ) of sugar beet as affected by the interactions of sowing dates (d) and potassium (K), in 2018/2019 season (Mean \pm SE) under soil salinity $\left(\mathrm{ECe}=8.96 \mathrm{dS} \mathrm{m}^{-1}\right)$ conditions, Figure S15: R-KUE $\left(\mathrm{kg} \mathrm{root} \mathrm{kg} \mathrm{K}^{-1}\right)$ and R-SUE $\left(\mathrm{kg} \mathrm{root} \mathrm{kg} \mathrm{S}^{-1}\right)$ of sugar beet as affected by the interactions of sowing dates (d) and potassium (K), in 2019/2020 season (Mean $\pm \mathrm{SE}$ ) under soil salinity $\left(\mathrm{ECe}=8.96 \mathrm{dS} \mathrm{m}^{-1}\right)$ conditions, Figure S16: R-KUE $\left(\mathrm{kg} \mathrm{root} \mathrm{kg} \mathrm{K}^{-1}\right)$ and 
R-SUE ( $\mathrm{kg}$ root $\mathrm{kg} \mathrm{S}^{-1}$ ) of sugar beet as affected by the interactions of sowing dates (d) and sulphure (S), in 2018/2019 season (Mean $\pm \mathrm{SE}$ ) under soil salinity $\left(\mathrm{ECe}=8.96 \mathrm{dS} \mathrm{m}^{-1}\right)$ conditions, Figure S17: R-KUE

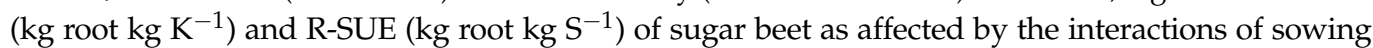
dates (d) and sulphure (S), in 2019/2020 season (Mean \pm SE) under soil salinity $\left(E C e=8.96 \mathrm{dS} \mathrm{m}^{-1}\right.$ )

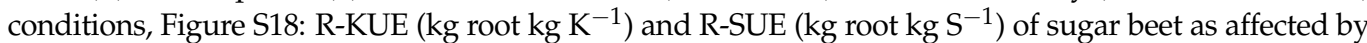
the interactions of potassium (K) and sulphur (S), in 2018/2019 season (Mean \pm SE) under soil salinity $\left(\mathrm{ECe}=8.96 \mathrm{dS} \mathrm{m}^{-1}\right)$ conditions, Figure S19: R-KUE $\left(\mathrm{kg}\right.$ root $\left.\mathrm{kg} \mathrm{K}^{-1}\right)$ and R-SUE $\left(\mathrm{kg}\right.$ root kg S $\left.\mathrm{kg}^{-1}\right)$ of sugar beet as affected by the interactions of potassium (K) and sulphur (S), in 2019/2020 season (Mean $\pm \mathrm{SE})$ under soil salinity $\left(\mathrm{ECe}=8.96 \mathrm{dS} \mathrm{m}^{-1}\right)$ conditions, Figure S20: Root yield $\left(\mathrm{Mg} \mathrm{ha}^{-1}\right)$ and pure sugar yield ( $\left.\mathrm{Mg} \mathrm{ha}^{-1}\right)$ of sugar beet as affected by the interactions of sowing date (D), potassium (K) and sulphur (S), in 2018/2019 season (Mean \pm SE) under soil salinity $\left(E C e=8.96 \mathrm{dS} \mathrm{m}^{-1}\right)$ conditions, Figure S21: Root yield $\left(\mathrm{Mg} \mathrm{ha}^{-1}\right)$ and pure sugar yield $\left(\mathrm{Mg} \mathrm{ha}^{-1}\right)$ of sugar beet as affected by the interactions of sowing date (D), potassium (K) and sulphur (S), in 2019/2020 season (Mean \pm SE) under soil salinity $\left(\mathrm{ECe}=8.96 \mathrm{dS} \mathrm{m}^{-1}\right)$ conditions.

Author Contributions: Conceptualization, A.A.A.M., M.M.R. and E.F.A.; data curation, A.A.A.M., M.M.R. and F.A.S.H.; formal analysis, A.A.A.M., E.F.A. and F.A.S.H.; investigation, A.A.A.M., M.M.R. and E.F.A.; methodology, A.A.A.M., M.M.R. and E.F.A.; resources, A.A.A.M., M.M.R., E.F.A. and F.A.S.H.; software, A.A.A.M., M.M.R. and F.A.S.H.; writing—original draft, A.A.A.M. and F.A.S.H.; writing-review and editing, M.M.R. and E.F.A. All authors have read and agreed to the published version of the manuscript.

Funding: The Deanship of Scientific Research at Taif University through the research number TURSP$2020 / 143$ is acknowledged.

Institutional Review Board Statement: Not applicable.

Informed Consent Statement: Not applicable.

Data Availability Statement: The data presented in this study are available upon request from the corresponding author.

Acknowledgments: Authors are thankful to Taif University Researchers Supporting Project number (TURSP-2020/143), Taif University, Taif, Saudi Arabia for providing the financial support and research facilities.

Conflicts of Interest: The authors declare no conflict of interest.

\section{References}

1. Desoky, E.M.; Merwad, A.M.; Rady, M.M. Natural biostimulants improve saline soil characteristics and salt stressed-sorghum performance. Commun. Soil Sci. Plant Anal. 2018, 49, 967-983. [CrossRef]

2. Rady, M.M.; Kuşvuran, A.; Alharby, H.F.; Alahrani, Y.; Kuşvuran, S. Pretreatment with proline or an organic bio-stimulant induces salt tolerance in wheat plants by improving antioxidant redox state and enzymatic activities and reducing the oxidative stress. J. Plant Growth Regul. 2019, 38, 449-462. [CrossRef]

3. Taha, R.S.; Seleiman, M.F.; Alotaibi, M.; Alhammad, B.A.; Rady, M.M.; Mahdi, A.H.A. Exogenous Potassium Treatments Elevate Salt Tolerance and Performances of Glycine max L. by Boosting Antioxidant Defense System under Actual Saline Field Conditions. Agronomy 2020, 10, 1741. [CrossRef]

4. Seleiman, M.F.; Semida, W.M.; Rady, M.M.; Mohamed, G.F.; Hemida, K.A.; Alhammad, B.A.; Hassan, M.M.; Shami, A. Sequential Application of Antioxidants Rectifies Ion Imbalance and Strengthens Antioxidant Systems in Salt-Stressed Cucumber. Plants 2020, 9, 1783. [CrossRef] [PubMed]

5. Zhang, P.; Yang, F.; Zhang, H.; Liu, L.; Liu, X.; Chen, J.; Wang, X.; Wang, Y.; Li, C. Beneficial Effects of Biochar-Based Organic Fertilizer on Nitrogen Assimilation, Antioxidant Capacities, and Photosynthesis of Sugar Beet (Beta vulgaris L.) under SalineAlkaline Stress. Agronomy 2020, 10, 1562. [CrossRef]

6. Koyro, H.-W. Effect of Salinity on Growth, Photosynthesis, Water Relations and Solute Composition of the Potential Cash Crop Halophyte Plantago coronopus (L.). Environ. Exp. Bot. 2006, 56, 136-146. [CrossRef]

7. Desoky, E.M.; EL-Maghraby, L.M.M.; Awad, A.E.; Abdo, A.I.; Rady, M.M.; Semida, W.M. Fennel and ammi seed extracts modulate antioxidant defence system and alleviate salinity stress in cowpea (Vigna unguiculata). Sci. Hortic. 2020, 272, 109576. [CrossRef]

8. ElSayed, A.I.; Boulila, M.; Rafudeen, M.S.; Mohamed, A.H.; Sengupta, S.; Rady, M.M.; Omar, A.A. Melatonin Regulatory Mechanisms and Phylogenetic Analyses of Melatonin Biosynthesis Related Genes Extracted from Peanut under Salinity Stress. Plants 2020, 9, 854. [CrossRef] 
9. Rehman, H.; Alharby, H.F.; Bamagoos, A.A.; Abdelhamid, M.T.; Rady, M.M. Sequenced application of glutathione as an antioxidant with an organic biostimulant improves physiological and metabolic adaptation to salinity in wheat. Plant Physiol. Biochem. 2021, 158, 43-52. [CrossRef]

10. Khan, A.H.; Ashraf, M.Y.; Naqvi, S.S.M.; Khanzada, B.; Ali, M. Growth and ion and solute contents of sorghum grown under $\mathrm{NaCl}$ and $\mathrm{Na}_{2} \mathrm{SO}_{4}$ salinity stress. Acta Physiol. Plant. 1995, 17, 261-268.

11. Illkaee, M.N.; Babaei, Z.; Baghdadi, A.; Golzardi, F. Effect of different planting dates and defoliation on the properties of sugar beet (Beta vulgaris L.). J. Exp. Biol. Agric. Sci. 2016, 4, 52-58. [CrossRef]

12. Pavlů, K.; Chochola, J.; Pulkrábek, J.; Urban, J. Influence of sowing and harvest dates on production of two different cultivars of sugar beet. Plant Soil Environ. 2017, 63, 76-81. [CrossRef]

13. Gobarah, M.E.; Hussein, M.M.; Tawfik, M.M.; Ahmed, A.G.; Mohamed, M.F. Effect of different sowing dates on quantity and quality of some promising sugar beet (Beta vulgaris L.) varieties under North Delta, condition. Egypt. J. Agron. 2019, 41, 343-354. [CrossRef]

14. Petkeviciene, B. The effect of climate factors on sugar beet early sowing timing. Agron. Res. 2009, 7, 436-443.

15. Mahdi, N.; Ali, A.; Ali, F. Study of effect of sowing and harvest date on sugar beet quantity and quality traits. Inter. J. Agron. Plant Prod. 2013, 4, 3392-3395.

16. Barłóg, P.; Grzebisz, W.; Feć, M.; Łukowiak, R.; Szczepaniak, W. Row method of sugar beet (Beta vulgaris L.) with multicomponent fertilizer based on urea-ammonium nitrate solution as a way to increase nutrient use efficiency. J. Cent. Eur. Agric. 2010, 11, 225-234.

17. Grzebisz, W.; Diatta, J. Constraints and solutions to maintain soil productivity: A case study from central Europe. In Soil Fertility Improvement and Integrated Nutrient Management-A Global Perspective; Whalen, J., Ed.; In Tech Europe: Rijeka, Croatia, 2012; pp. 159-182.

18. Tzortzakis, N.G. Influence of $\mathrm{NaCl}$ and calcium foliar spray on lettuce and endive growth using nutrient film technique. Int. J. Veg. Sci. 2009, 15, 1-13. [CrossRef]

19. Marschner, H. Mineral Nutrition of Higher Plants; Academic Press: Cambridge, MA, USA, 1995.

20. Fathy, M.F.; Abdel- Motagally, I.; Kamal, K.A. Response of Sugar Beet Plants to Nitrogen and Potassium Fertilization in Sandy Calcareous Soil. Int. J. Agric. Biol. 2009, 11, 695-700.

21. Mehrandish, M.; Moeini, M.J.; Armin, M. Sugar beet (Beta vulgaris L.) response to potassium application under full and deficit irrigation. Eur. J. Exp. Biol. 2012, 2, 2113-2119.

22. Wang, M.; Zheng, Q.; Shen, Q.; Guo, S. The critical role of potassium in plant stress response. Int. J. Mol. Sci. 2013, 14, 7370-7390. [CrossRef]

23. Liu, Y.; Tc-Zou, G.; Geng, X.-F.; Wang, Y. Effect of potash on sugar beet quality and adversity resistance. China-Sugar Beet. 1992, 4, 15-20.

24. Khalil, S.M.; Mostafa, S.N.; Mostafa, Z.R. Influence of potassium fertilizer and soil salinity on chemical composition of sugar beet root. Minufiya J. Agric. Res. 2001, 26, 583-594.

25. Hussain, Z.; Khattak, R.A.; Irshad, M.; Mahmood, Q. Sugar beet (Beta vulgaris L.) response to diammonium phosphate and potassium sulphate under saline-sodic conditions. Soil Use Manag. 2014, 30, 320-327. [CrossRef]

26. Merwad, A.M.A. Efficiency of potassium fertilization and salicylic acid on yield and nutrient accumulation of sugar beet grown on saline soil. Commun. Soil Sci. Plant Anal. 2016, 47, 1184-1192. [CrossRef]

27. Osman, A.S.; Rady, M.M. Ameliorative effects of sulphur and humic acid on the growth, antioxidant levels, and yields of pea (Pisum sativum L.) plants grown in reclaimed saline soil. J. Hortic. Sci. Biotechnol. 2012, 87, 626-632. [CrossRef]

28. Mekdad, A.A.A.; Rady, M.M. Response of Beta vulgaris L. to nitrogen and micronutrients in dry environment. Plant Soil Environ. 2016, 62, 23-29.

29. Willenbrink, J. Über Beziehungen zwischen Proteinumsatz und Schwefelversorgung der Chloroplasten. Z. Pflanzenphysiol. 1967, $56,427-438$.

30. Thomas, S.G.; Bilsborrow, P.E.; Hocking, T.J.; Bennett, J. Effect of sulphur deficiency on the growth and metabolism of sugar beet (Beta vulgaris cv Druid). J. Sci. Food Agric. 2000, 80, 2057-2062. [CrossRef]

31. Hashem, F.; El- Maghraby, S.; Wassif, M. Efficiency of organic manure and residual sulphur under saline irrigation water and calcareous soil conditions. Egypt. J. Soil. Sci. 1997, 37, 451-465.

32. Kastori, R.; Plescnicar, M.; Arsenijevic-Maksimovic, I.; Petrovic, N.; Pankovic, D.; Sakac, Z. Photosynthesis, chlorophyll fluorescence and water relations of young sugar beet plants as affected by sulfur supply. J. Plant Nutr. 2000, 23, 1037-1049. [CrossRef]

33. Thomas, S.G.; Hocking, T.J.; Bilsborrow, P.E. Effect of sulphur fertilization on the growth and metabolism of sugar beet grown on soils of differing sulphur status. Field Crops Res. 2003, 83, 223-235. [CrossRef]

34. Awad, N.M.M.; Gharib, H.S.; Moustafa, S.M.I. Response of sugar beet (Beta vulgaris L.) to potassium and sulphur supply in clayed soil at north delta, Egypt. Egypt. J. Agron. 2013, 35, 77-99. [CrossRef]

35. Tawfic, S.F.; Abdel Aziz, R.M.; Eanar, A.K. Effect of planting date and sulphur fertilizer on yield and quality of sugar beet under newly reclaimed soils. J. Plant Prod. Mansoura Univ. 2014, 5, 1547-1556. [CrossRef]

36. Ponce, V.M.; Pandey, R.P.; Ercan, S. Characterization of drought across the climate spectrum. J. Hydrol. Eng. 2000, 5, 222-224. [CrossRef] 
37. Page, A.I.; Miller, R.H.; Keeny, D.R. Methods of Soil Analysis. In Part II: Chemical and Microbiological Methods, 2nd ed.; American Society of Agronomy: Madison, WI, USA, 1982; pp. 225-246.

38. Soil Survey Staff USDA. Soil Taxonomy: A Basic System of Soil Classification for Making sand Interpreting Soil Surveys, 2nd ed.; Agriculture Handbook No. 466; USDA: Washington, DC, USA, 1999.

39. Milford, G.F.J.; Pocock, T.O.; Jaggard, K.W.; Biscoe, P.V.; Armstrong, M.J.; Last, P.J. An analysis of leaf growth in sugar beet. IV. The expansion of the leaf canopy in relation to temperature and nitrogen. Ann. Appl. Biol. 1985, 107, 335-347. [CrossRef]

40. Spoustová, P.; Synková, H.; Valcke, R.; `Ce ${ }^{\smile r o v s k a ́, ~ N . ~ C h l o r o p h y l l ~ a ~ F l u o r e s c e n c e ~ a s ~ a ~ t o o l ~ f o r ~ a ~ s t u d y ~ o f ~ t h e ~ P o t a t o ~ v i r u s ~ Y ~}$ effects on photosynthesis of nontransgenic and transgenic Pssuipt tobacco. Photosynthetica 2013, 51, 191-201. [CrossRef]

41. Clark, A.J.; Landolt, W.; Bucher, J.B.; Strasser, R.J. Beech (Fagus sylvatica) response to ozone exposure assessed with a chlorophyll a fluorescence performance index. Environ. Pollut. 2000, 109, 501-507. [CrossRef]

42. Watson, D.J. The physiological basis of variation in yield. In Advances in Agronomy; Academic Press: Cambridge, MA, USA, 1952; Volume 665, pp. 101-145.

43. McGinnus, R.A. Sugar Beet Technology, 2nd ed.; Sugar Beet Development Foundation: Denver, CO, USA, 1971.

44. Harvey, G.W.; Dotton, J.V. Root quality and processing. In The Sugar Beet Crop Science into Practice; Cooke, D.A., Scott, R.K., Eds.; Chapman \& Hall: London, UK, 1993; pp. 571-617.

45. FAO. Crop Water Information: Sugar Beet. 2012. Available online: http://www.fao.org/nr/water/cropinfo_sugarbeet.html (accessed on 1 February 2012).

46. Nikpanah, H.; Seifzadeh, S.; Hemayati, S.S.; Shiranirad, A.; Taleghani, D. Effects of management of agronomical factors on sugar beet steckling production and growth. Int. J. Bioinf. Res. Appl. 2015, 7, 959-964.

47. Ribeiro, R.V.; Machado, E.C.; de Oliveira, R.F. Temperature response of photosynthesis and its interaction with light intensity in sweet orange leaf discs under non-photorespiratory condition. Ciênc. Agrotecnol. 2006, 30, 670-678. [CrossRef]

48. El-Hag, M.A.; Ahmed, A.O.; Ragga, P.W.M. Evaluation of sowing date and harvesting ages of some sugar beet (Beta vulgaris L.) cultivars under Guneid condition (Sudan). Int. J. Agri. Res. Rev. 2015, 3, 421-424.

49. Al-Jbawi, E.M.; Al-Zubi, H.I. Effect of sowing date and length of storage on storability in sugar beets (Beta vulgaris L.) piles. Scholarly. J. Agri. Sci. 2016, 6, 25-31.

50. Hatam, Z.; Sabet, M.S.; Malakouti, M.J.; Mokhtassi-Bidgoli, A.; Homaee, M. Zinc and potassium fertilizer recommendation for cotton seedlings under salinity stress based on gas exchange and chlorophyll fluorescence responses. S. Afr. J. Bot. 2020, 130, 155-164. [CrossRef]

51. Hermans, C.; Hammond, J.P.; White, P.J.; Verbruggen, N. How to plants respond to nutrient shortage by biomass allocation? Trends Plant Sci. 2006, 11, 610-617. [CrossRef] [PubMed]

52. Zhang, L.; Gao, M.; Li, S.; Alva, A.K.; Ashraf, M. Potassium fertilization mitigates the adverse effects of drought on selected Zea mays cultivars. Turk. J. Bot. 2014, 38, 713-723. [CrossRef]

53. Chen, J.; Guo, Z.; Chen, H.; Yang, X.; Geng, J. Effects of different potassium fertilizer types and dosages on cotton yield, soil available potassium and leaf photosynthesis. Arch. Agron. Soil Sci. 2020. [CrossRef]

54. Nitoses, R.E.; Evans, H.G. Effect of univalent cations on the activity of particulate starch synthase. Plant Physiol. 1969, 44, 1260-1266. [CrossRef] [PubMed]

55. Draycott, A.P. Sugar Beet; Blackwell Publishing Ltd.: Oxford, UK, 2006; 474p.

56. Al-Elwany, O.A.A.I.; Mohamed, G.F.; Abdurrahman, H.A.; Rady, M.M.; Latef, A.A.A. Exogenous glutathione-mediated tolerance to deficit irrigation in salt-affected Capsicum frutescence (L.) plants is connected with higher antioxidant content and ionic homeostasis. Not. Bot. Horti Agrobot. Cluj-Napoca 2020, 48, 1957-1979. [CrossRef]

57. Desoky, E.S.; Mansour, E.; Ali, M.M.A.; Yasin, M.A.T.; Abdul-Hamid, M.I.E.; Rady, M.M.; Ali, E.F. Exogenously used 24epibrassinolide promotes drought tolerance in maize hybrids by improving plant and water productivity in an arid environment. Plants 2021, 10, 354. [CrossRef]

58. Semida, W.M.; Abdelkhalik, A.; Mohamed, G.F.; Abd El-Mageed, T.A.; Abd El-Mageed, S.A.; Rady, M.M.; Ali, E.F. Foliar Application of Zinc Oxide Nanoparticles Promotes Drought Stress Tolerance in Eggplant (Solanum melongena L.). Plants 2021, 10, 421. [CrossRef]

59. Rady, M.M.; Boriek, S.H.K.; Abd El-Mageed, T.A.; Seif El-Yazal, M.A.; Ali, E.F.; Hassan, F.A.S.; Abdelkhalik, A. Exogenous Gibberellic Acid or Dilute Bee Honey Boosts Drought Stress Tolerance in Vicia faba by Rebalancing Osmoprotectants, Antioxidants, Nutrients, and Phytohormones. Plants 2021, 10, 748. [CrossRef] 